\title{
Modelo de gestión para el control de riesgos en oleoductos, poliductos y gasoductos
}

Alfonso Eduardo Rivas Tufiño ${ }^{1}$

Sergio Julio NúÑEZ Solano ${ }^{2}$ Ronal Elicio Moscoso JÁcOME ${ }^{3}$

ReCibido: 25/10/2019 Aceptado: 06/11/2019 Publicado: 16/10/2020

\begin{abstract}
RESUMEN
La industria del transporte de hidrocarburos combina sus actividades técnicas con la integridad de los sistemas operativos instalados, además de la implementación de la seguridad de los ductos ante fallas y fugas de líquidos peligrosos. Surge, así, el modelo de gestión para el control de riesgos en todos los procesos de envío de productos, en el que se alinea la configuración de los estándares técnicos para operar los ductos en cumplimiento de las condiciones y acciones subestándar, que derivan a la obligatoriedad de mantener la integridad (capacidad instalada). Ello da origen al nivel de gestión del control de riesgo, como alcance del objetivo al nivel máximo de conformidad de las instalaciones, lo que asegura a todas las unidades operativas alcanzar el desarrollo de nuevos conceptos de trabajo proactivo, y no reactivo, ante los siniestros no planificados. En este estudio, se logró la reducción de los niveles de riesgo significativo del $30.16 \%$ al $10.26 \%$, al corte del periodo 2014-2017, lo cual se refleja en una disminución en los efectos de daños en las instalaciones, reducción de las compensaciones extrajudiciales y operación amigable con el ambiente en concordancia con las demandas planificadas en el giro del negocio.
\end{abstract}

Palabras clave: modelos de gestión; control de riesgos; interacciones; condiciones.

\section{INTRODUCCIÓN}

En las supervisiones especiales llevadas a cabo en el Oleoducto Norperuano del 27 al 29 de enero del 2016 y del 6 al 11 de febrero del 2016, se verificó que en la progresiva del PK 440+781 del tramo II, así como en la progresiva del punto kilométrico 206+031 del Ramal Norte, el desbordamiento de petróleo crudo se produjo por falla en la tubería a efectos de corrosión externa. Ello implica que se configuró un alto riesgo de impacto negativo no solo al suelo y a las quebradas Inayo y Cashacaño, y a los ríos Chiriaco y Morona, sino también a la vida y la salud de las personas que habitan en las zonas aledañas a los derrames (Organismo de Evaluación y Fiscalización Ambiental, 2016).

De otro lado, en abril de 2013, se reportó un derrame de 5500 barriles de crudo en el Oleoducto de Crudos Pesados (OCP). Con respecto a dicho desastre, Alexandra Almeida, activista de la Fundación Acción Ecológica y entrevistada para la nota del diario La Hora, señaló que los efectos ambientales fueron nefastos sobre todo en las fuentes hídricas cercanas: "Es sorprende que sea del OCP porque es relativamente nuevo. Nos dijeron que era a prueba de movimiento sísmico que no se iba a romper" (La Hora, 9 de abril de 2013, párr. 11).

1 Ingeniero industrial por la Universidad de Guayaquil (Guayaquil, Ecuador), diplomado en Ingeniería del Fuego por The University of Texas System (Austin, Estados Unidos), magíster en Seguridad y Prevención de Riesgos del Trabajo por la Universidad Tecnológica Equinoccial (Quito, Ecuador), doctorado en Ingeniería Industrial por la Universidad Nacional Mayor de San Marcos (Lima, Perú) e instructor NFPA 1041. Posee más de 16 años de experiencia en el sector petrolero y en el desarrollo de poliductos y oleoductos con seguridad industrial, salud ocupacional y protección al medio ambiente. Actualmente, labora en Petroecuador como supervisor de Seguridad, Salud y Ambiente. (Santo Domingo, Ecuador). ORCID: https://orcid.org/0000-0001-8188-268X

E-mail: alfonso.rivas@eppetroecuador.ec, almirante20039@hotmail.com

2 Ingeniero químico por la Escuela Superior Politécnica de Chimborazo (Riobamba, Ecuador), magíster en Sistemas Integrados de Gestión de Calidad, Ambiente y Seguridad por la Universidad Politécnica Salesiana (Guayaquil, Ecuador), doctorado en Ingeniería Industrial por la Universidad Nacional Mayor de San Marcos (Lima, Perú) y auditor ISO 9001, 14001, 22001 y OHSAS 18001. Posee más de 12 años de experiencia en empresas nacionales y multinacionales en producción, seguridad, salud y medio ambiente. Actualmente, es consultor independiente. (Riobamba, Ecuador)

ORCID: https://orcid.org/0000-0001-8804-3088

E-mail: sergio.nunez@nestle.com.ec

3 Ingeniero de mantenimiento por la Escuela Superior Politécnica de Chimborazo (Riobamba, Ecuador), magíster en Educación Superior y doctorado en Ingeniería Industrial por la Universidad Nacional Mayor de San Marcos (Lima, Perú). Actualmente, es coordinador de la estación de bombeo y representante de la dirección en las normas ISO 14001-2004 de la Estación Corazón, encargado de la repotenciación del Poliducto Esmeraldas-Quito. (Quito, Ecuador).

ORCID: https://orcid.org/0000-0001-6890-4039

E-mail: ronal.moscoso@eppetroecuador.ec 
Finalmente, el mismo diario, el 26 de febrero de 2005, recordaba el derrame de crudo y posterior incendio en la refinería de Esmeraldas ocurrida en 1998:

Escenas de dolor y angustia fueron las que vivió la ciudad de Esmeraldas y en particular, las familias asentadas en las riberas de los ríos Teaone y Esmeraldas, al verse amenazadas por el incendio ocurrido la noche del jueves 26 de febrero de 1998, hace siete años.

Eran las 21 h00 cuando el pánico se apoderó de toda la población, tras la ruptura del Sistema del Oleoducto Trans Ecuatoriano (SOTE) a la altura de las villas de Petroecuador, provocando un incendio que dejó 4 muertos.

De acuerdo con los datos entregados por la Defensa Civil, 60 personas resultaron quemadas, 6 fueron reportadas como desaparecidas; 120 casas se quemaron, 20 quedaron semidestruidas y 10 fibras fueron dañadas por el fuego (párrs. 1-3).

En ese contexto, se puede afirmar que la asignación (o reasignación) de recursos para la reducción de la probabilidad de falla es, comúnmente, la forma más efectiva de practicar la gestión del control de riesgos (Muhlbauer, 2004).

El tema de esta investigación surgió a raíz del derrame de 1998, tras presenciar la catástrofe más grande en la historia de Esmeraldas-Ecuador, causada por fallas geológicas y como resultado de la fractura mecánica de los ductos empaquetadas de petróleo y el otro de gasolina. Ello originó la fuga de productos de forma continua con destino al cuerpo receptor hídrico "río" y la emisión de gases inflamables. Así, después de encontrar una fuente de ignición desconocida, se generó una reacción en cadena y una explosión que culminó con la pérdida de vidas humanas y una trágica consecuencia ambiental en la flora y fauna del río Teaona.

Esta tragedia se identificó como una consecuencia de la falta de inspección y toma de acciones correctivas de conformidad técnica específica en geomática y mantenimiento de línea, al no optar con el pilotaje y anclaje de las dos estructuras de marco $\mathrm{H}$. Si esto se hubiera tomado en cuenta, se podría haber prevenido esta catástrofe ambiental y humana. Guy y Mohamed (2007) indican que los desperfectos externos, como la corrosión, los rasguños de objetos extraños y las actividades de montaje de tuberías, son las razones principales para el fallo de tuberías.

En la actualidad, las empresas petroleras buscan implementar en sus instalaciones nuevas tecnologías de última generación, de innovación y control, con avances importantes para la seguridad en el transporte de hidrocarburos por ductos, ratificando los eventos continuos en las operaciones de carga de estos compuestos. Ello ha demostrado que los desarrollos tecnológicos son importantes en la línea del flujo y el control de transferencias de productos; no obstante, la tecnología de innovación no interviene en el examen del conjunto del universo de riesgos que producen fugas y contaminación. Se observa, en la Figura 1, el producto de acciones y/o condiciones "antes, durante y después" de fallas materializadas. La tecnología instalada en los procesos no define la alerta temprana de riesgos incubados, "micro y macro", en su evolución desde el origen con la probabilidad indeterminada por el tiempo y espacio que demande la materialización de los hechos. En estas condiciones, la gestión del control de riesgo por fugas y derrames de hidrocarburos en los poliductos y gasoductos se determina por la alerta temprana previa a la materialización de los eventos.

La monitorización de fluidos y procesos proporciona información sobre los parámetros que pueden causar una alerta temprana para remediar la corrosión interna. Dichos parámetros deben identificarse para cada gasoducto y deben incluir el monitoreo de los métodos de mitigación químicos y físicos (Kermani y Chevrot, 2012).

El objetivo planteado es identificar los niveles de gestión del riesgo corporativo que generan estados de no conformidad en las instalaciones de transporte, con influencias determinadas por etapas (suelo, "gestión"; ductos, "corrosión") de integridad al flujo continuo en líneas. El modelo de gestión del control de riesgos se constituyó en un objetivo de cotejo técnico a la identificación, monitoreo, vigilancia, medición y divulgación de los hallazgos incubados a la inseguridad corporativa. Se debe cumplir con la búsqueda de reducir siniestros no deseados que afecten a las comunidades aledañas, las líneas del transporte de hidrocarburos, el ambiente, el derecho de vía frente a los cruces con las tuberías empaquetadas de productos peligrosos.

\section{METODOLOGÍA}

En la investigación de fallas de ductos con la data cuantitativa que generó la inspección interna y externa, mediante el uso de sonda de FML a 2.5 


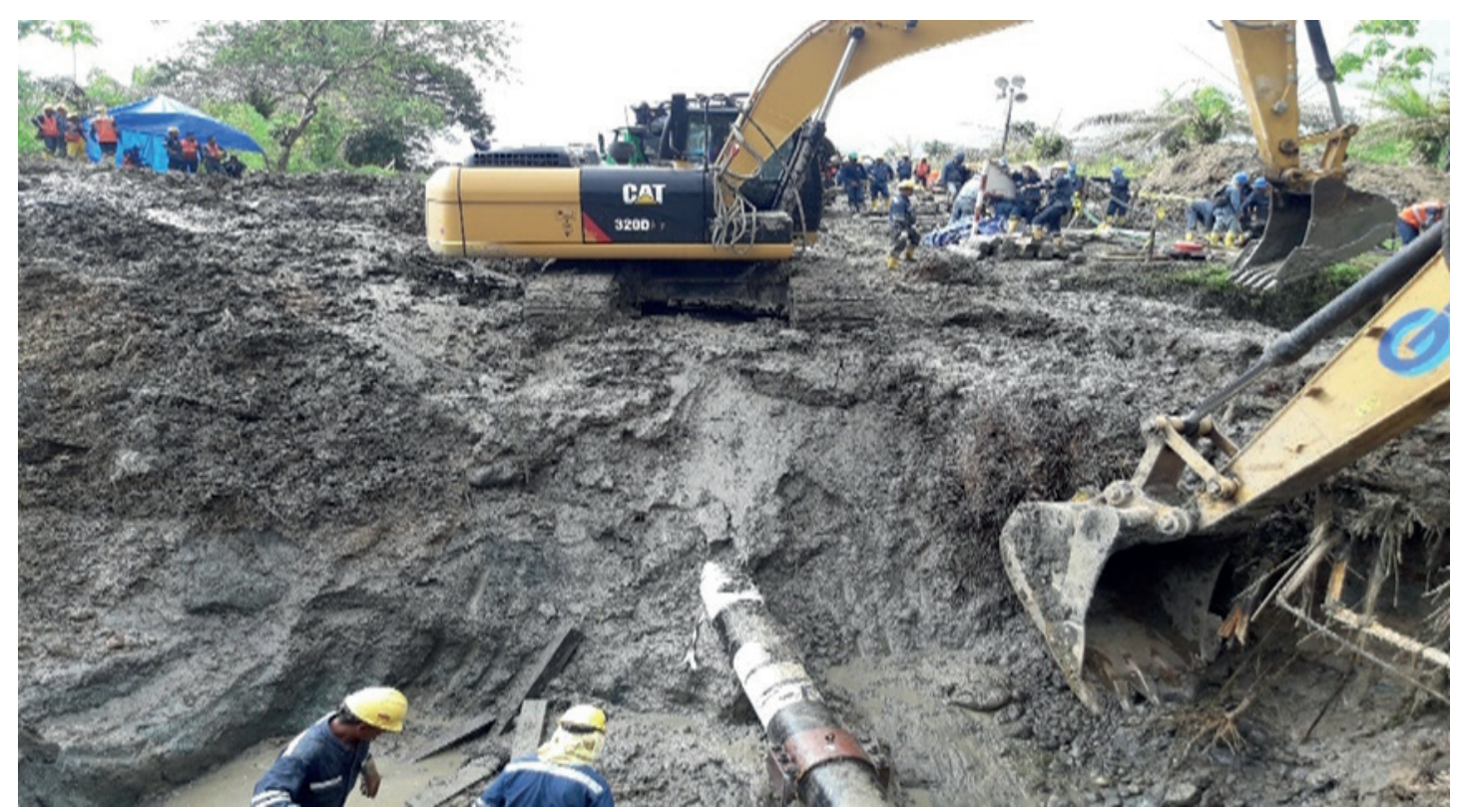

Figura 1. Rotura de ducto del Poliducto Esmeraldas-Santo Domingo, Ecuador. Fuente: Petroecuador.

$\mathrm{km} / \mathrm{h}$, se identificaron los determinantes de riesgos del km 0 al km 164 del Poliducto de Petroecuador de Esmeradas Santo Domingo. Se desarrolló la investigación aplicativa para la generación de conocimiento y el diseño experimental de manipulación de variables independientes. Se observaron los efectos que producen las variables dependientes.

El nuevo paradigma puede cambiar muchos hábitos, cultura y prácticas útiles a tener en cuenta. Varias propiedades ocultas aparecen cuando se comparan con una aproximación clásica, se vinculan fuertemente en un nuevo paradigma de gestión del control del riesgo y las partes técnicas se interesan por aplicarlo (Massotte y Corsi, 2015).

1. Exploratorio: identificar y monitorear los determinantes de fallas para hallar los fenómenos con alto y bajo nivel de probabilidad de riesgos incubados (micro y macro).

2. Descriptivo: especificar los determinantes de criticidad en el origen del riesgo.

3. Correlacional: asociar las variables del estado de gestión y conformidad.

4. Explicativo: entender el fenómeno de incertidumbre y/o acertar la gestión como resultado obtenido de minimizar los impactos de fallas, fuga y derrame, y contaminación ambiental.
Existen varios tipos de encuestas: inspección externa, evaluación directa o en línea, inspecciones usando la fuga de flujo magnético (MFL) o equipo ultrasónico. Todas ellas generan información detallada sobre la condición de la tubería y el alcance de anomalías y corrosión. Esta información guía el funcionamiento de las tuberías o define las actividades de mantenimiento (Adam y Davis, 2009).

\section{Identificación y determinación del riesgo}

Al contrastar la información de acciones y condiciones subestándar, la relación de causa-efecto del riesgo se determina en la fuente, medio o receptor entre el ducto y los derechos de vía.

Se comprobó que existen mayores accidentes por deslaves en el sistema del Oleoducto Transecuatoriano y poliductos con propiedades cuantitativas sobre la mecánica de criticidad de fallas geológicas del suelo, uno de los motivos de la fuga de hidrocarburos. Ello se observa en la Figura 2.

Para Bai y Bai (2014), cada segmento de tubería tiene su propio riesgo, como la producción de probabilidad de falla y consecuencia de falla.

Las condiciones subestándares de alto riesgo identificadas a lo largo de los $164 \mathrm{~km}$ de longitud del DdV (derecho de vía) del poliducto y oleoducto de Esmeraldas-Santo Domingo son: 


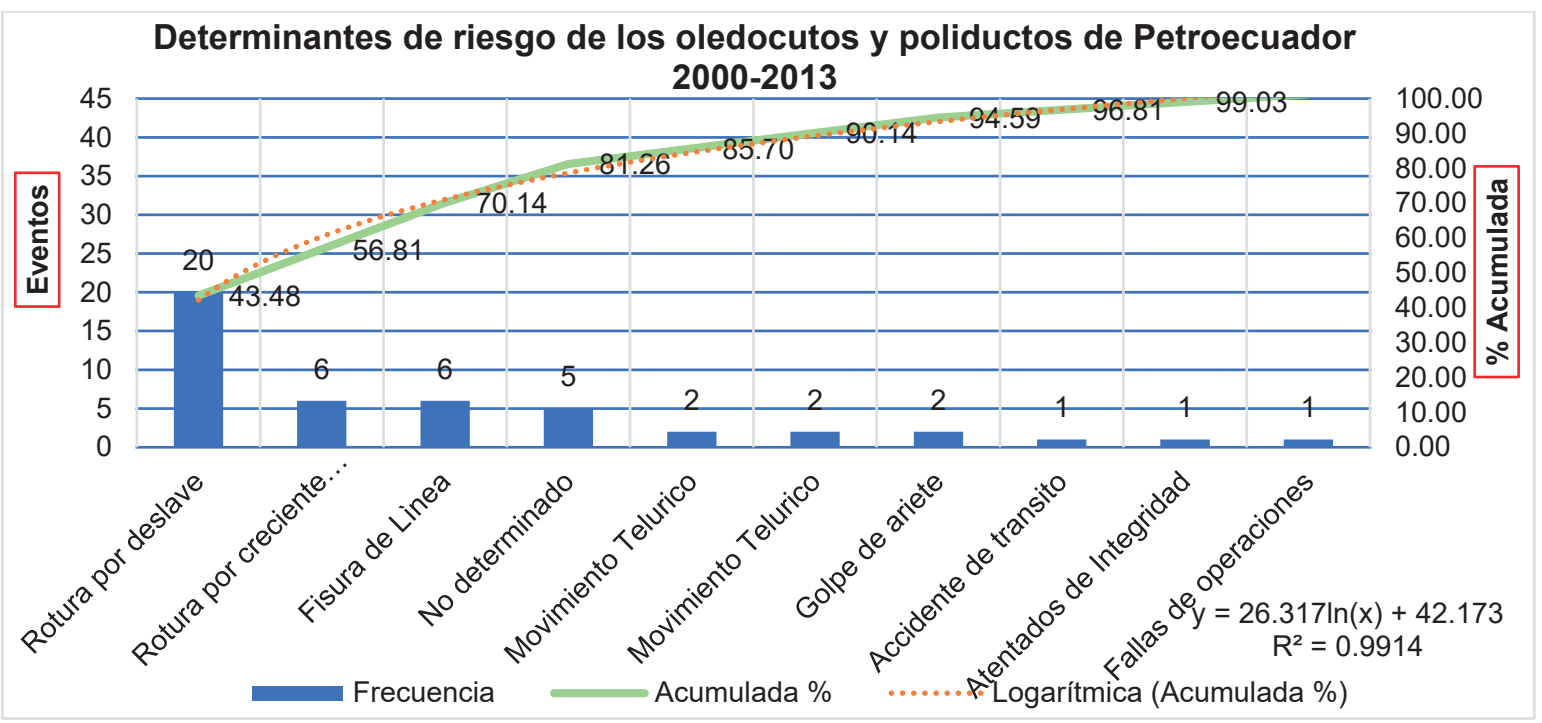

Figura 2. Determinantes de riesgo y probabilidad de falla, fuga con derrames hidrocarburos. Fuente: Petroecuador.

- Deslizamientos y/o movimientos de tierra superficiales.

- Deslizamientos y/o movimientos de tierra profundos.

- Hundimiento paulatino del terreno.

- Falla superficial y desclasamiento en el substrato rocoso.

- Erosión fluvial en los estratos blandos ubicados en los márgenes del DdV y desmoronamiento de estratos más resistentes.

- Sitios de erosión que activan los taludes y provocan colapsos en los muros de contención.

- Señalización de puntos de arrastre de tubería por deslaves-soterramiento.

- Punto "apantallamiento-pilotear falla".

- Accidentes geográficos del derecho de vía y sus influencias sísmicas.

- Altimetría de perfil del ducto y derecho de vía (trazo de variantes)

- Tasa demográfica.

- Interferencia con límites de propiedad, calles y caminos, guarniciones y todos los datos que ayuden a definir claramente el derecho de vía con identificación a falla de diaclasas.

- Incorporación de mapas de incidencia de la región; topográficos, hidrográficos, geológicos, etc.
- Desarrollo del perfil altimétrico, zonas pobladas y válvulas del ducto.

- Fotografías aéreas; podrá ser conveniente en caso de no contarse con mapas o cartas.

- Análisis por geo-tag y por división sectorial.

- Identificación de la influencia del paso de los ductos con la división sectorial por el Instituto Geográfico Militar.

- Determinación de las cartas de uso del suelo, normalmente editadas por el Instituto Nacional de Estadística, Geografía e Informática (INEGI). Estas cartas permitirán definir posibles afectaciones sobre el derecho de vía propuesto o instalado.

\section{El modelo de gestión de control de riesgo}

Precisa y emplea la integridad y la confiabilidad de los ductos con intervención del mantenimiento de línea; otorga relevancia en la sostenibilidad del transporte de hidrocarburos y derecho de vía.

- Integridad y confiabilidad. Caracterización del suelo del derecho de vía.

- Identifica el tipo de suelos para el mejoramiento de la conductividad de protección catódica del ducto.

- Puntos de interferencias de corrientes parásitas que afectan a la integridad externa del ducto.

- Herramientas de limpieza interna. 
- Herramientas de inspección (transmisor/ receptor de marcación, ultrasonido, magnetic flux leakage).

- Resultados de resistividad del suelo/ducto.

- Análisis hidrodinámicos en derecho de vía.

- Puntos de control de fuga de hidrocarburos.

- Monitoreo de flujo de magnetismo-corriente impresa.

- Dosificación e inyección de inhibidor de corrosión.

- Monitoreo de puntos de pérdidas de espesores y sus relaciones de progresiva.

- Recorridos y análisis de datos campo de condiciones e informes de resultados.

Induce, también, a la preparación organizativa y técnica en detalle de la tubería requerida para posibilitar el lanzamiento y la recepción de herramientas inteligentes, lo que garantiza su paso seguro y la obtención de los datos más exactos sobre el estado de corrosión (Timashev y Bushinskaya, 2016).

\section{Incidencia ON-OFF/Natural en derecho de vía}

- Ensayar los contenidos de cloruros en el suelo.

- Revisar y comprobar la resistividad del terreno.

- Inspeccionar la cercanía a líneas de transmisión eléctrica de alto voltaje.

- Inspeccionar potenciales variables entre las tuberías.

- Intervenir en los suelos afectados por corrientes parásitas.

- Inspeccionar los cruces con otras líneas de interconexión eléctrica o estructural.

- Verificar históricos por 3 años.
- Verificar $850 \mathrm{mv}=>$ Pot. ON $<=2100 \mathrm{mv}$ (trimestral).

- Verificar si por $\mathrm{ON}<850$, verificar procedimiento de prueba.

- Verificar la norma de los 100 mv.

Podríamos cuantificar las pérdidas presentes en todo sistema de transporte de hidrocarburos, como los costos de interrupción del servicio, el costo del producto perdido, el costo de limpieza, entre otras. Las consecuencias a veces se agrupan en categorías directas e indirectas, donde los costos directos son incluyentes de la integridad e influyen en los procesos del control de riesgos frente a las fugas de hidrocarburos (Muhlbauer, 2004).

\section{Resultados obtenidos de la inspección interna y externa}

Se ejecutó el envío de la sonda inteligente de flujo magnético en línea (FML). Se observaron anomalías de corrosión internas y externas, como se puede apreciar en los resultados del coeficiente de integridad como indicador (ver Figura 3), con determinación de fallas mayores por corrosión externas, mas hay un predominio alto de riesgo significativo de anomalías incubadas al coeficiente $<1$, como valor crítico en relación al ancho de profundidad de la anomalía de corrosión y el nominal de diseño con la máxima presión segura de operación calculada sobre la presión de descarga del flujo continuo.

La corrosión puede definirse como un deterioro de un metal debido a reacciones químicas o electroquímicas entre el metal y su medio ambiente. La tendencia de un metal a corroerse depende de un ambiente determinado y del tipo de metal. La presencia de dióxido de carbono $\left(\mathrm{CO}_{2}\right)$, sulfuro de hidrógeno $\left(\mathrm{H}_{2} \mathrm{~S}\right)$ y agua libre en el fluido de producción puede causar graves problemas de corrosión (Bai y Bai, 2014).

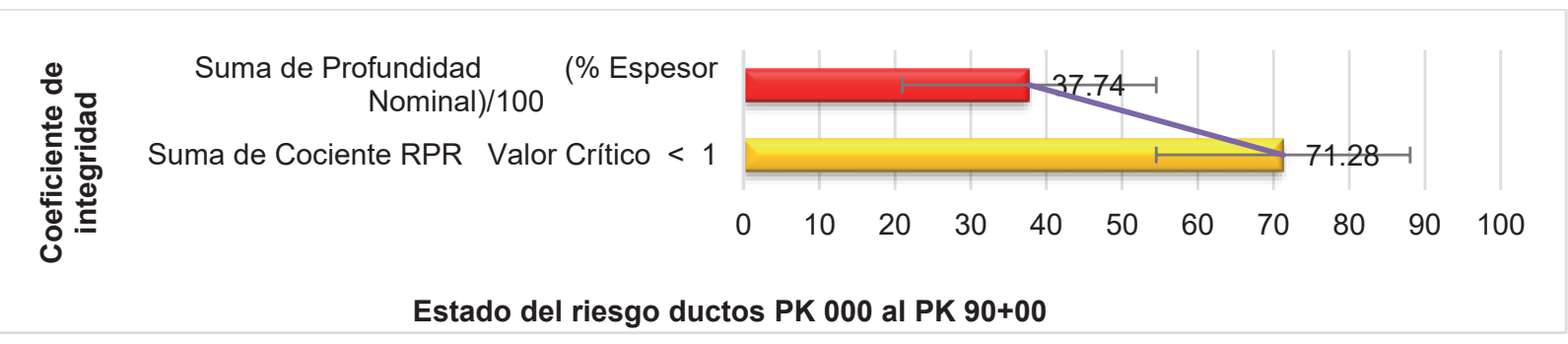

Figura 3. Estado del control de riesgo en la corrosión del Poliducto Esmeraldas-Santo Domingo, año 2014 (punto km 000 al punto $\mathrm{km} \mathrm{90).}$

Fuente. Petroecuador. Inspección interna y externa Poliducto Esmeraldas-Santo Domingo de 2014. 
Ahora, se presenta el estado del control de riesgo del Poliducto Esmeraldas-Santo Domingo en el año 2017. Se puede observar una mejora significativa del corte del 2017 (ver Figura 4) en relación al 2014 (ver Figura 3).

\section{Utilizar y sostener}

La gestión asegura la estructura durante el tiempo de operaciones y la vincula al desarrollo y la innovación de propuestas encaminadas a reducir los siniestros que generan los ductos; de este modo, se busca identificar la probabilidad de falla mediante el análisis de las condiciones y acciones que interfieren a lo largo del derecho de vía de los 164 kilómetros del Poliducto y Oleoducto del Esmeraldas-Santo Domingo, para lo cual se toma en cuenta una muestra (riesgo interno del punto kilométrico 050 al punto kilométrico 96).

A partir de la muestra, se desarrollaron: práctica de prestaciones de cotejo técnico con estándares de operación y control; requisitos de normativas (ver Tabla 1); códigos de prácticas de inspección, mantenimiento, calificación, reparación y alteración; soldadura predictiva; prácticas de eventos;

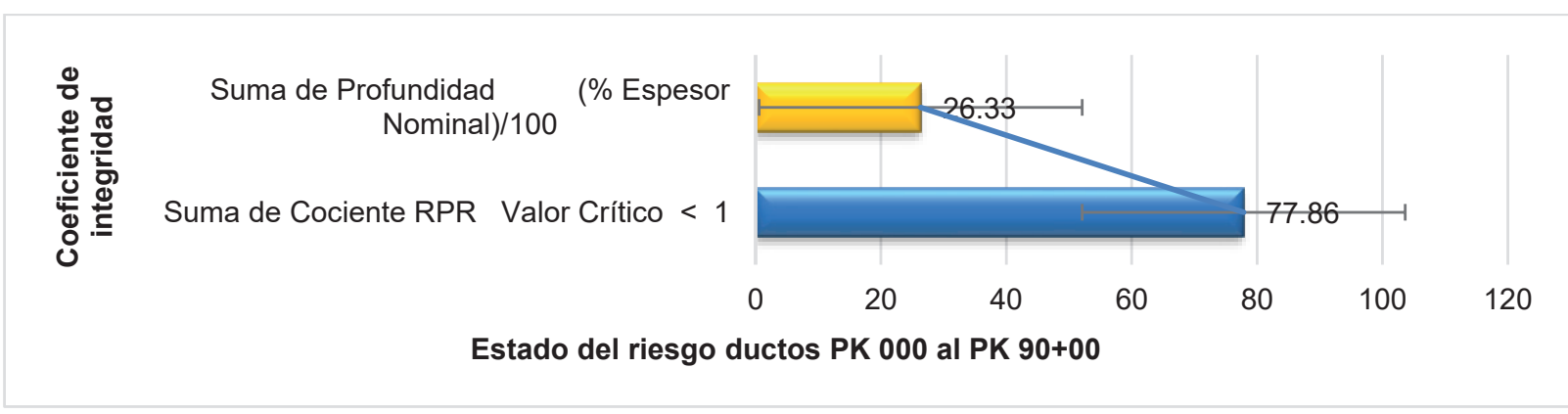

Figura 4. Estado del control riesgo en la corrosión del Poliducto Esmeraldas-Santo Domingo, año 2017 (punto km 000 al punto $\mathrm{km} \mathrm{90)}$.

Fuente. Petroecuador. Inspección interna y externa Poliducto Esmeraldas-Santo Domingo de 2017.

Tabla 1. Instalaciones y su conformidad operativa.

\begin{tabular}{|c|c|c|c|c|c|}
\hline \multicolumn{6}{|c|}{ Niveles de gestión: Control de riesgo $(\mathrm{N}+, \mathrm{N}-; \mathrm{C})$ / Estado de conformidad } \\
\hline $\begin{array}{c}\# \\
\text { Inst. }\end{array}$ & \multicolumn{3}{|c|}{$\begin{array}{c}\text { Instalaciones: estados de conformidad } \\
\text { / integridad y confiabilidad }\end{array}$} & \multicolumn{2}{|r|}{ Unidades operativas } \\
\hline 1 & \multicolumn{3}{|l|}{ Ductos } & \multirow{4}{*}{\multicolumn{2}{|c|}{$\begin{array}{l}\text { UGO 1: Mantenimiento de línea del sistema de transporte de hidrocarburos } \\
\text { UGO 4: Integridad y confiabilidad del transporte de hidrocarburos } \\
\text { UGO 7: Seguridad física "Patrullas militares y personal civil" } \\
\text { UGO 5: Geomática para el derecho de vía y ductos del transporte de hidrocarburos }\end{array}$}} \\
\hline 2 & Rectificador & & & & \\
\hline 3 & \multirow{2}{*}{\multicolumn{3}{|c|}{ Derecho de vía }} & & \\
\hline 4 & & & & & \\
\hline 5 & \multicolumn{3}{|c|}{ Estación de bombeo } & \multirow{3}{*}{\multicolumn{2}{|c|}{$\begin{array}{l}\text { UGO 2: Operaciones I } \\
\text { UGO 3: Operaciones II } \\
\text { UGO 6: Laboratorio }\end{array}$}} \\
\hline 6 & \multicolumn{3}{|c|}{ Granjas de tanques de estación } & & \\
\hline 7 & \multicolumn{3}{|c|}{ Manifold lanzamiento de herramientas } & & \\
\hline 8 & \multicolumn{3}{|c|}{ Válvulas de control en línea } & \multicolumn{2}{|c|}{ UGO 8: Mantenimiento electromecánico de estado de bombeo } \\
\hline C & NC_neg & NC_pos & $\% \mathrm{C}$ & Periodo: & Unidades: análisis integridad \\
\hline 34 & 0 & 0 & & \multirow{9}{*}{ Mes/año } & 34 \\
\hline 42 & 0 & 0 & & & 42 \\
\hline 8 & 0 & 0 & & & 8 \\
\hline 24 & 0 & 0 & & & 24 \\
\hline 6 & 0 & 0 & & & 6 \\
\hline 13 & 0 & 0 & & & 13 \\
\hline 11 & 0 & 0 & & & 11 \\
\hline 8 & 0 & 0 & & & 8 \\
\hline 146 & 0 & 0 & & & 146 \\
\hline
\end{tabular}

UGO: unidades de gestión operativa.

Fuente: Elaboración propia. 
prácticas recomendadas; prácticas seguras; prácticas de instrumentación; pruebas destructivas; ensayos no destructivos; mantenimiento impreso de cama tierra profunda; práctica soldadura de tubería manual para determinar la resistencia y métodos para evaluar la aceptación de defectos en estructuras de metal.

Dichos parámetros deben identificarse para cada gasoducto y debe incluirse el monitoreo de los métodos de mitigación químicos y físicos (Kermani y Chevrot, 2012).

Se identifica el desempeño de la integridad operativo, reconociendo conformidad por el porcentaje del acatamiento de estándares en relación al instrumento de cotejo técnico para verificar el cumplimiento del plan operativo enfocando a las no conformidades mayores, no conformidades menores y conformidad del control de los riesgos sincronizados en la confiabilidad del transporte de hidrocarburos.

\section{Fórmula de conformidad ALRI}

La fórmula de conformidad ALRI identifica el estado de la gestión en las instalaciones:

$$
\mathrm{C}=\left(\mathrm{C} / \Sigma \mathrm{C}+\mathrm{N}_{-}+\mathrm{N}+\right) 1
$$

Donde:

C: = Conformidad; cumplimiento de un requisito convertido en actividad.

$\mathrm{N}_{-}=$No conformidad menor; falta de actualización e implementación de procedimiento.

$\mathrm{N}+=$ No conformidad mayor; falta del cumplimiento del procedimiento al control de evento.

\section{$1=$ Constante .}

Se obtiene el nivel de gestión de control de riesgo presente del año 2014, con el máximo no permisible de 2.654 punto de criticidad, lo que marca su estado de vulnerabilidad a la topografía de fallas, fugas y derrames de consideración a la inseguridad estimada por las unidades operativas de los oleoductos, poliductos y gasoductos. Se utiliza el instrumento de cotejo en referencia (ver Tabla 2).

La concentración de variables independientes genera determinantes de modos de falla en los factores adyacentes al diseño de puesta en marcha del sistema de instalaciones de transporte de hidrocarburos, en el que se presenta probabilidad de sucesos (riesgos incubados) que desencadenan en eventos no deseados con nivel de riesgo en 2.51 puntos, que representa condiciones inseguras (validez baja) durante el periodo del año 2014, lo que señala una desmejorada gestión sobre la base de 10 puntos de la validez de gestión perfecta, lo cual es reflejado por 8 componentes (unidades operativas), observados en la Tabla 3.

La matriz de cotejo técnico mejoró la confiabilidad y la integridad de las unidades operativas; además, logró consolidar la progresiva de gestión e incidir significativamente al corte del año 2017 sobre la base de 6.08 , en relación a la verificación de cumplimiento técnico del suelo, ductos y flujo por tuberías con incidencia en la gestión permanente de alcanzar la integridad y confiabilidad del transporte de hidrocarburos seguros y minimizar los impactos ambientales que se generan, como riesgos incubados macros.

El nivel de riesgo por cumplimiento de cotejo técnico aplicando a la correlación de los años 2014-2017

Tabla 2. Unidades de gestión del control del riesgo por unidades de análisis.

\begin{tabular}{|l|c|}
\hline Unidades operativas de oleoductos, poliductos y gasoductos & Unidades de análisis \\
\hline UGO 1: Mantenimiento de línea del sistema de transporte de hidrocarburos & 34 \\
\hline UGO 2: Operaciones I & 13 \\
\hline UGO 3: Operaciones II & 6 \\
\hline UGO 4: Integridad y confiabilidad del transporte de hidrocarburos & 42 \\
\hline UGO 5: Geomática para el derecho de vía y ductos del transporte de hidrocarburos & 24 \\
\hline UGO 6: Laboratorio & 11 \\
\hline UGO 7: Seguridad física "Patrullas militares y personal civil" & 8 \\
\hline UGO 8. Mantenimiento electromecánico de estado de bombeo & 8 \\
\hline Total & 146 \\
\hline
\end{tabular}

Fuente: Elaboración propia. 
por el número de unidades de análisis con responsabilidad corporativa del transporte hidrocarburos se puede observar en la Figura 5.

Se evidencia que el nivel de control de riesgos es significativo de 5-10\% al corte del año 2017.

\section{Fórmula de nivel de control de gestión/riesgo operativo por acotejo técnico}

$$
\begin{gathered}
\mathrm{Ng}=\text { VUG1+VUG2+VUG3+... VUG8 } \\
\mathrm{Ng}=(\mathrm{Na} / \mathrm{Va} * \mathrm{Vn}) * 10
\end{gathered}
$$

Donde:

VUG = Valor total de acciones $/$ unidad de análisis.

$\mathrm{Na}=$ Número de actividades .

$\mathrm{Ng}=$ Nivel de gestión de riesgo .
$\mathrm{Vn}=$ Valor nominal

Va $=$ Valor de unidades de análisis

\section{RESULTADOS}

\section{Resultado 1}

El enfoque de gestión tradicional hacia un cambio de gestión basada por identificación, monitoreo, control, medición y divulgación de los riesgos nos permite mantener la continuidad del negocio del transporte de los hidrocarburos con una reducción de las fugas de las líneas en operaciones amigables con el medio ambiente.

La tabla 4 muestra la diferencia entre el modelo tradicional y el nuevo modelo de enfoque para evaluar el riesgo y la gestión de control de condiciones no planificadas en ductos de hidrocarburos. Según la

Tabla 3. Indicadores de gestión del control de riesgo sobre la base de 1 a 10.

\begin{tabular}{|c|c|c|c|c|}
\hline \multirow{2}{*}{ Unidades de gestión } & $\mathbf{2 0 1 4}$ & $\mathbf{2 0 1 5}$ & $\mathbf{2 0 1 6}$ & $\mathbf{2 0 1 7}$ \\
\cline { 2 - 5 } & X_Punto UG /10 & X_Punto UG /10 & X_Punto UG /10 & X_Punto UG /10 \\
\hline UGO 1 & 3.29 & 4.14 & 4.82 & 6.35 \\
\hline UGO 2 & 3.88 & 4.10 & 4.74 & 7.50 \\
\hline UGO 3 & 1.53 & 2.50 & 3.06 & 8.00 \\
\hline UGO 4 & 3.17 & 3.33 & 4.68 & 6.27 \\
\hline UGO 5 & 1.67 & 2.75 & 3.44 & 5.11 \\
\hline UGO 6 & 1.00 & 1.82 & 3.48 & 3.64 \\
\hline UGO 7 & 3.75 & 4.17 & 6.80 & 8.00 \\
\hline UGO 8 & 1.80 & 2.76 & 2.85 & 3.78 \\
\hline Total, Riesgo UG & 2.51 & 3.20 & 4.23 & 6.08 \\
\hline
\end{tabular}

Fuente. Elaboración propia.

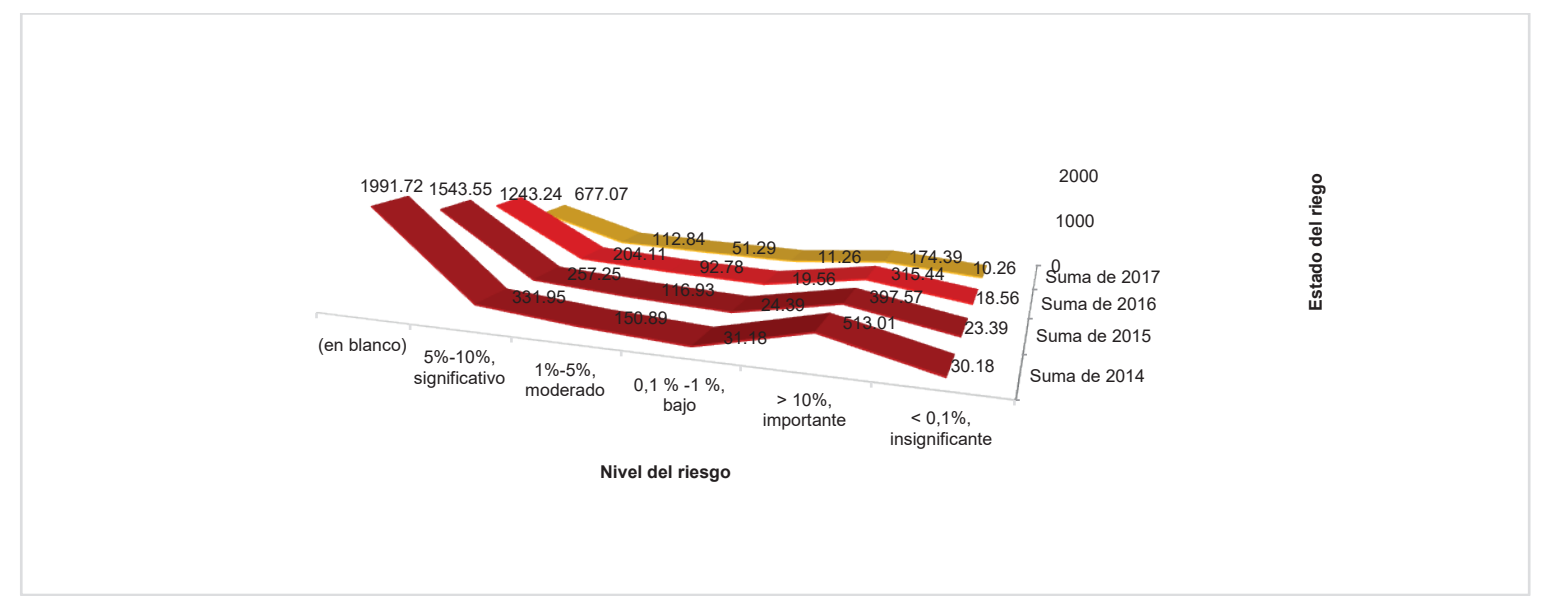

Figura 5. Estado del nivel de control del riesgo Poliducto Esmeraldas-Santo Domingo en los años 2014-2017.

Fuente. Elaboración propia. 
tendencia, se evidencia en los poliductos y oleoductos Esmeraldas-Quito la reducción de eventos de 3.5 por año a 1 evento por cada año.

En la Figura 6, se evidencia la representación del resultado obtenido en la implementación del Modelo de Gestión del Poliducto Esmeraldas-Santo Domingo al corte del año 2017.

En el sentido de la gestión eficaz del riesgo, los resultados se garantizan en concordancia con los objetivos estratégicos de la organización de Petroecuador; con esta visión, la gestión integral del control de riesgo se vuelve el factor clave en el éxito de reducir las pérdidas de línea y la contaminación del ambiente. Los sistemas de gestión son «estructuras probadas para la gestión y mejora continua de las políticas, los procedimientos y procesos de la organización» (SIGWEB, 2016). Estos sistemas pueden ser individuales o de interacciones complejas.

\section{Resultado 2}

Capacidad instalada de 30660000 bls./año. El rendimiento al corte del periodo 2014 al 2017 en barriles de hidrocarburos transportados por Petroecuador Poliducto Esmeraldas-Santo Domingo con tendencia significativa en el año 2018:

Año 2014: 22505273 bls./año transportados. 72\% inicio de gestión del control del riesgo.

Año 2017: 24077474 bls./año transportados. $78.53 \%$, resultados esperados.

Año 2018: 25523034 bls./año transportados. $83.25 \%$ tendencia de integridad y confiabilidad.

Lineamentos del modelo de gestión y control del riesgo:

a. Dispone criterios de intervención ante oportunidades de mejora y el dominio del control de riesgo en las unidades de gestión operativa y conformidad de instalaciones en procesos de transporte de hidrocarburos; los cuales dependerán de estrategias y métodos técnicos para la intervención, como planes de sostenibilidad de negocio en relación a los resultados esperados (cero siniestros).

Tabla 4. Líneas de gestión de conformidad anual comparativa del control del riesgo en ductos de hidrocarburos.

\begin{tabular}{|c|c|c|c|}
\hline Etiquetas de fila & Suma de C & Suma de NC_neg & Suma de NC_pos \\
\hline 2014 & 16 & 41 & 89 \\
\hline 2015 & 40 & 29 & 47 \\
\hline 2016 & 65 & 37 & 15 \\
\hline 2017 & 101 & 22 & 2 \\
\hline Año & 2 & 4 & 227 \\
\hline Total general & 224 & 133 & 44 \\
\hline
\end{tabular}

C: Conformidad, NC_neg: no conformidad menor, NC_pos: no conformidad mayor.

Fuente. Elaboración propia.

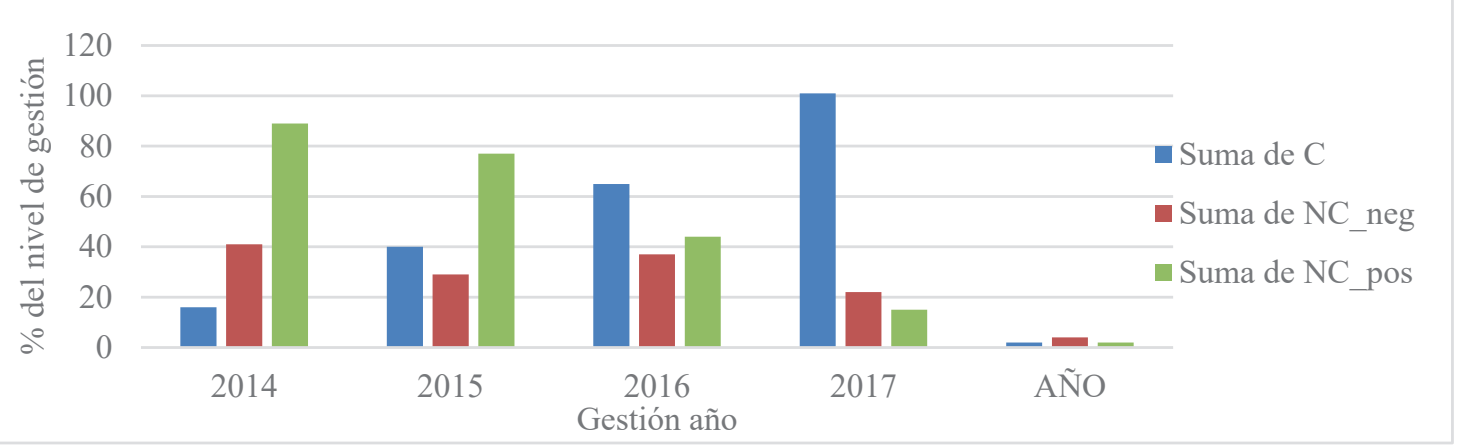

Figura 6. Gestión del control de riesgo en la conformidad funcional del Poliducto Esmeraldas-Santo Domingo de los años 2014-2017, Petroecuador.

Fuente. Elaboración propia. 
b. Requiere un mapa de riesgo, áreas de exposición a fugas con incendio y explosión; en consecuencia, establece el riesgo máximo aceptable, así como el área no aceptable.

c. Emplea la medición del monitoreo-categorías de riesgo con probabilidad de causar daños al proceso del transporte de hidrocarburos por ductos y derecho de vía.

d. Utiliza la proyección del nivel de pérdida ante el monitoreo-medición de control permanente en la fuente, el medio y el receptor que originan pérdidas por causa y efecto de la materialización del siniestro.

e. Precisa los mecanismos de proyección de máxima cobertura de contingencia frente al riesgo de fuga de hidrocarburos.

f. Emplea, a lo largo del derecho de vía, la cobertura permanente de alerta temprana al peligro y riesgo con probabilidad de siniestros en pobladores aledaños del transporte de hidrocarburos.

g. La gestión define y considera medidas de desempeño a la identificación ajustada contra el riesgo a lo largo del derecho de vía y ductos.

\section{Resultado 3}

Se consolidaron las estructuras del diseño del modelo de gestión para obtener el producto final de la organización en un software (ver Figura 7). Se presenta el manejo de aplicaciones desarrolladas e incorporadas a las unidades operativas del mandato al control de riesgo, operando desde la base del sistema corporativo con ingreso y validación de la información ejecutada en campo.

El programador de control de riesgo puede ayudar a cuantificar los equilibrios entre costo y seguridad en la etapa de diseño y, también, a identificar las políticas y los horarios para inspecciones con pruebas rentables, mantenimiento preventivo, provisión de repuestos, asignación de redundancia y reemplazo de partes de trabajo para mantener los sistemas complejos operando, según lo previsto, a lo largo de su vida de diseño (Cox, 2009).

\section{DISCUSIÓN}

El modelo de gestión para el control de riesgo para las empresas petroleras permite influir en las empresas corredoras de pólizas de seguros de la industria del petróleo. Al tomar posesión de los activos, no se

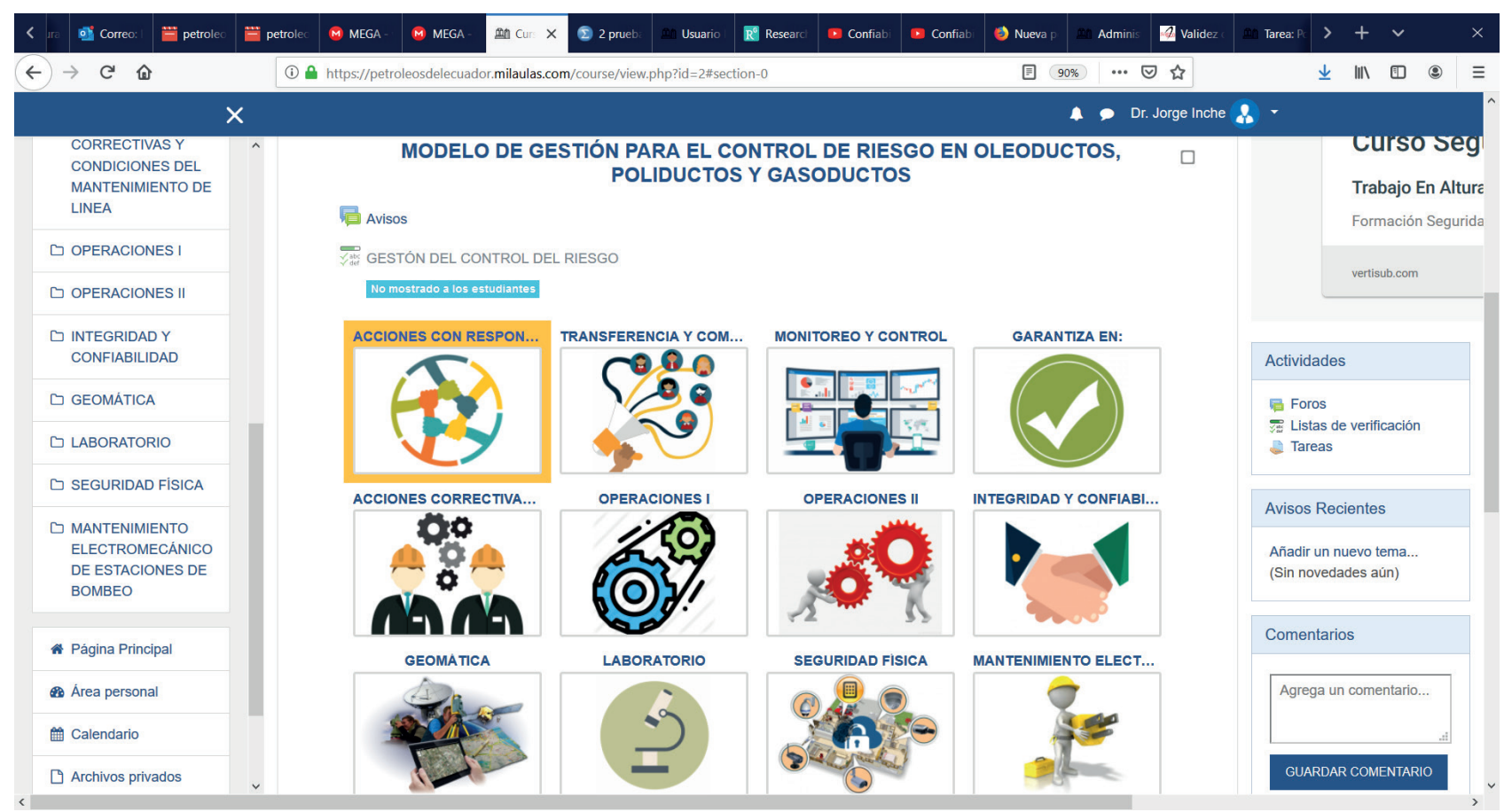

Figura 7. Modelo de gestión operativo desde la plataforma corporativa.

Fuente: Elaboración propia. Software modelo de gestión para el control de riesgo del transporte de hidrocarburos. 
busca eliminar los riesgos, sino la gestión eficiente por parte de la entidad aseguradora, para lo cual se necesita identificarlos, medirlos y controlarlos, así la prevención de riesgos y el modelo establecido en las unidades de gestión operativa trabajan de forma integral y no por separado.

\section{CONCLUSIÓN}

Se encontraron evidencias suficientes en los niveles del estado de riesgo para afirmar que el modelo de gestión del control de eventos corporativos del transporte de hidrocarburos con puntaje de las unidades operativas de gestión influye significativamente en los porcentajes de conformidad de las instalaciones (flujo de hidrocarburos, ducto de tuberías y suelo derecho de vía).

- La corrosión pasó a ser controlada en el cumplimiento de los estándares de integridad y confiabilidad de los ductos (anomalías externas e internas); con el cociente RPR valor crítico < 1 se controló el estado del nivel del riesgo de los ductos y, como resultado, la producción se incrementó.

- Con la máxima operación permisible, en el año 2014, la máxima presión de operación fue de 1050 psi y 2600 bls./hora; en el 2018, la carga subió a 1200 psi y 3200 bls./hora en gasolinas y 3000 bls./hora con diésel petrolero, por lo que se cumplió con la proyección de la demanda estimada, utilizando la capacidad instalada.

- Se minimizaron los daños ambientales y se aprovechó la capacidad instalada del transporte de hidrocarburos del periodo 2014, que era $72 \%$; del 2017 , que era $78.53 \%$; y del 2018 , que al corte era más de $83.25 \%$.

\section{AGRADECIMIENTO}

Un profundo agradecimiento a las unidades operativas del Poliducto Esmeraldas-Santo Domingo-Quito de Petroecuador, por las facilidades en favor de la investigación y desarrollo, que vinculamos permanentemente a la prevención de riesgos, con miras trazadas a reducir los impactos ambientales y mejorar la confiabilidad y la integridad de los procesos operativos del transporte de hidrocarburos. Se realiza esta investigación en cumplimiento del cotejo técnico estandarizado que, además, se constituye en una práctica amigable con el ambiente y comunidades aledañas al derecho de vías.

\section{REFERENCIAS BIBLIOGRÁFICAS}

[1] Adam, S. y Davis, K. (2009). Pipeline Geomatics. Practice and Innovation. Fairfield, Estados Unidos: American Society of Mechanical Engineers.

[2] Bai, Y. y Bai, Q. (2014). Subsea Pipeline Integrity and Risk Management. Massachusetts, Estados Unidos: Gulf Professional Publishing.

[3] Cox, A. (2009). Risk Analysis of Complex and Uncertain Systems. Nueva York, Estados Unidos: Springer.

[4] Guy, P. y Elwany, M. H. (2007). Safety, Reliability and Risks Associated with Water, Oil and Gas Pipelines. Heidelberg, Alemania: Springer.

[5] Kermani, B. y Chevrot, T. (2012). Recommended Practice for Corrosion Management of Pipelines in Oil and Gas Production and Transportation. Leeds, Reino Unido: Maney Publishing / European Federation of Corrosion.

[6] La Hora (26 de febrero de 2005). Hace siete años Esmeraldas vivió una noche de terror. La Hora. Recuperado de https://lahora. com.ec/noticia/1000310432/hace-siete-aosesmeraldas-vivi-una-noche-de-terror.

[7] La Hora (9 de abril de 2013). Derrame de 5.500 barriles de crudo en Esmeraldas. La Hora. Recuperado de https://lahora.com.ec/ noticia/1101489994/derrame-de-5500-barrilesde-crudo-en-esmeraldas.

[8] Massotte, P. y Corsi, P. (2015). Operationalizing Sustainability. Londres, Reino Unido: Wiley / ISTE.

[9] Muhlbauer, W. K. (2004). Pipeline Risk Mangement Manual. Ideas, Techniques and Resources. Massachusetts, Estados Unidos: Elsevier.

[10] Organismo de Evaluación y Fiscalización Ambiental (2016). Resolución Directoral N. ${ }^{\circ}$ 12-2016-OEFA/DS. Informe N. ${ }^{\circ}$ 307-2016OEFA/DS-HID. Recuperado de http://www. oefa.gob.pe/wp-content/uploads/2019/03/ RESOLUCION-N012-2016-DS.pdf.

[11] SIGWEB (2016). Ventajas de los Sistemas de Gestión de Seguridad y Salud en Trabajo. SIGWEB. Recuperado de http://www.sigweb. cl/2016/10/20/sistemas-integrados-de-gestion.

[12] Timashev, S. y Bushinskaya., A. (2016). Diagnostics and Reliability of Pipeline Systems. Cham, Suiza: Springer. 


\section{Risk Control Management Model for Gas, Oil Pipelines and Polyducts}

Alfonso Eduardo Rivas Tufiño Sergio Julio NúÑ̃z Solano ${ }^{2}$ Ronal Elicio Moscoso JÁcOME ${ }^{3}$

\begin{abstract}
The hydrocarbon transport industry combines its technical activities with the integrity of the installed operating systems, in addition to the implementation of safety measures for pipelines against failures and leakage of dangerous liquids. Thus, the risk control management model in all product shipment processes arises, which aligns the configuration of technical standards to operate pipelines in compliance with the prevention of substandard conditions and actions, which derive from the obligation to maintain pipeline integrity (installed capacity). This gives origin to a certain level of risk control management, as an achievement of the maximum level of conformity with the facilities, which ensures that all operating units achieve the development of new concepts of proactive, not reactive, work, in the face of unplanned disasters. In this study, significant risk levels were reduced from $30.16 \%$ to $10.26 \%$ at the end of the 2014-2017 period, which is reflected in a decrease in the consequences of the damages in facilities, a reduction in out-of-court settlements and an increase in environmentally friendly operations in accordance with industry standards.
\end{abstract}

Keywords: management models; risk control; interactions; conditions.

\section{INTRODUCTION}

During the environmental post-incident monitoring conducted on the North Peruvian Pipeline from January 27 to 29, 2016 and February 6 to 11, 2016, it was verified that in KP 440+781 of section II, as well as in KP 206+031 of the North Branch, the overflow of crude oil had been produced by pipe failure due to external corrosion. This implies that there was a high risk of negative impact not only on the soil and on the Inayo and Cashacaño streams and the Chiriaco and Morona rivers, but also on the life and health of the people living in the areas surrounding the spills (Organismo de Evaluación y Fiscalización Ambiental, 2016).

On the other hand, in April 2013, a spill of 5,500 barrels of crude oil was reported in the Heavy Crude Oil Pipeline (OCP). In an interview for the newspaper La Hora, Alexandra Almeida, an activist from the Acción Ecológica Foundation, said that the environmental effects were disastrous, especially to the nearby water sources: "Es sorprende que sea del OCP porque es relativamente nuevo. Nos dijeron que era a prueba de movimiento sísmico que no se iba a romper" [It is surprising that it happened to the OCP as it is relatively new. They told us that it was earthquake

1 Industrial engineer from the Universidad de Guayaquil (Guayaquil, Ecuador), Diploma in Fire Engineering from the Universidad Tecnológica de La Habana José Antonio Echeverría. Master in Safety and Risk Prevention in the Workplace from Universidad Tecnológica Equinoccial (Quito, Ecuador) and PhD in Industrial Engineering from the Universidad Nacional Mayor de San Marcos (Lima, Peru). Over 16 years of experience in the oil sector and in the development of polyducts and oil pipelines with industrial safety, occupational health and environmental protection. Currently working as supervisor of Safety, Health and Environment at Petroecuador (Santo Domingo, Ecuador).

ORCID: https://orcid.org/0000-0001-8188-268X

E-mail: alfonso.rivas@eppetroecuador.ec, almirante20039@hotmail.com

2 Chemical engineer from the Escuela Superior Politécnica de Chimborazo (Riobamba, Ecuador), Master in Master in Integrated Systems of Quality, Environment and Safety Management from The Universidad Politécnica Salesiana (Guayaquil, Ecuador), $\mathrm{PhD}$ in Industrial Engineering from Universidad Nacional Mayor de San Marcos (Lima, Peru) and ISO 9001, 14001, 22001 and OHSAS 18001 standard auditor. Over 12 years of experience in national and multinational companies of production, safety, health and environment sectors. Currently working as an independent consultant. (Riobamba, Ecuador).

ORCID: https://orcid.org/0000-0001-8804-3088

E-mail: sergio.nunez@nestle.com.ec

3 Maintenance engineer from the Escuela Superior Politécnica de Chimborazo (Riobamba, Ecuador), Master in Higher Education and $\mathrm{PhD}$ in Industrial Engineering from the Universidad Nacional Mayor de San Marcos (Lima, Peru). Currently working as coordinator of the pumping station and management representative for ISO 14001-2004 standards at Corazón Station, in charge of the repowering of the Esmeraldas-Quito Pipeline. (Quito, Ecuador)

ORCID: https://orcid.org/0000-0001-6890-4039

E-mail: ronal.moscoso@eppetroecuador.ec 
proof and that it was not going to break] (La Hora, April 9, 2013, para. 11).

Finally, on February 26, 2005, the same newspaper recalled the oil spill and subsequent fire at Esmeraldas refinery in 1998:

\begin{abstract}
"Escenas de dolor y angustia fueron las que vivió la ciudad de Esmeraldas y en particular, las familias asentadas en las riberas de los ríos Teaone y Esmeraldas, al verse amenazadas por el incendio ocurrido la noche del jueves 26 de febrero de 1998, hace siete años.
\end{abstract}

Eran las 21 h00 cuando el pánico se apoderó de toda la población, tras la ruptura del Sistema del Oleoducto Trans Ecuatoriano (SOTE) a la altura de las villas de Petroecuador, provocando un incendio que dejó 4 muertos.

De acuerdo con los datos entregados por la Defensa Civil, 60 personas resultaron quemadas, 6 fueron reportadas como desaparecidas; 120 casas se quemaron, 20 quedaron semidestruidas y 10 fibras fueron dañadas por el fuego." [Scenes of pain and anguish were experienced in the city of Esmeraldas, particularly by the families settled on the Teaone and Esmeraldas riverbanks, when they were threatened by the fire that occurred on the night of Thursday, February 26, 1998, seven years ago. It was 9:00 p.m. when panic broke out among the entire population after the rupture of the Trans-Ecuadorian Oil Pipeline System (SOTE) near the Petroecuador settlements, causing a fire that left four dead. According to data provided by the Civil Defense, 60 people were burned, 6 were reported missing, 120 houses were burned, 20 were semi-destroyed and 10 fiberglass canoes were damaged by fire] (paras. 1-3).

In that context, it can be stated that the allocation (or reallocation) of resources for the reduction of failure probability is commonly the most effective way to practice risk control management (Muhlbauer, 2004).

The subject of this investigation arose from the spill of 1998, after witnessing the largest catastrophe in the history of Esmeraldas-Ecuador, caused by geological faults, which in turn caused the mechanical fracture of an oil and a gas pipeline. This produced the leakage of products in a continuous way to a waterbody, the river, and the emission of flammable gases. Thus, after finding an unknown ignition source, a chain reaction started followed by an explosion, culminating in the loss of human lives and a tragic environmental consequence on the flora and fauna of the Teaona River.

This tragedy was identified as a consequence of as the lack of inspection and lack of corrective actions according to specific and technical principles of geomatics and line maintenance, that is, by opting not to piledrive and anchor two $\mathrm{H}$-frame structures. If this had been taken into account, this environmental and human catastrophe could have been prevented. Guy and Mohamed (2007) indicate that external damage, such as corrosion, scratches from foreign objects and pipeline assembly activities are the main reasons for pipeline failure.

At present, oil companies are seeking to implement new state-of-the-art technologies in the areas of innovation and control, with important progress for hydrocarbon pipeline transportation security, emphasizing the continuous events along the load operations of these materials. This has demonstrated that technological developments are important in the flowline and product transfers control; however, innovation technology does not intervene in the evaluation of the risks regarding producing spills and contamination. Figure 1 shows the product of actions and/or conditions "before, during and after" materialized failures. The technology installed in the processes does not give an early warning of the evolution of cumulative risks (micro and macro cumulative risk) from the origin, so that the probability of risks is not available. In this context, risk control management for oil leaks and spills in polyducts and gas pipelines is determined by early warning prior to event occurrences.

The monitoring of fluids and processes provides information on the parameters that may provide early warning to remediate internal corrosion. Such parameters should be identified for each pipeline and should include monitoring of chemical and physical mitigation methods (Kermani \& Chevrot, 2012).

The objective is to identify the risk levels in corporate management causing the non-conformity states in the transportation facilities, whose influences are determined by levels of integrity (soil, "management"; pipelines, "corrosion") of the continuous flowlines. The risk control management model aimed to establish a technical comparison against the identification, monitoring, surveillance, measurement and disclosure of cumulative risks found in the corporate management model. The search for 


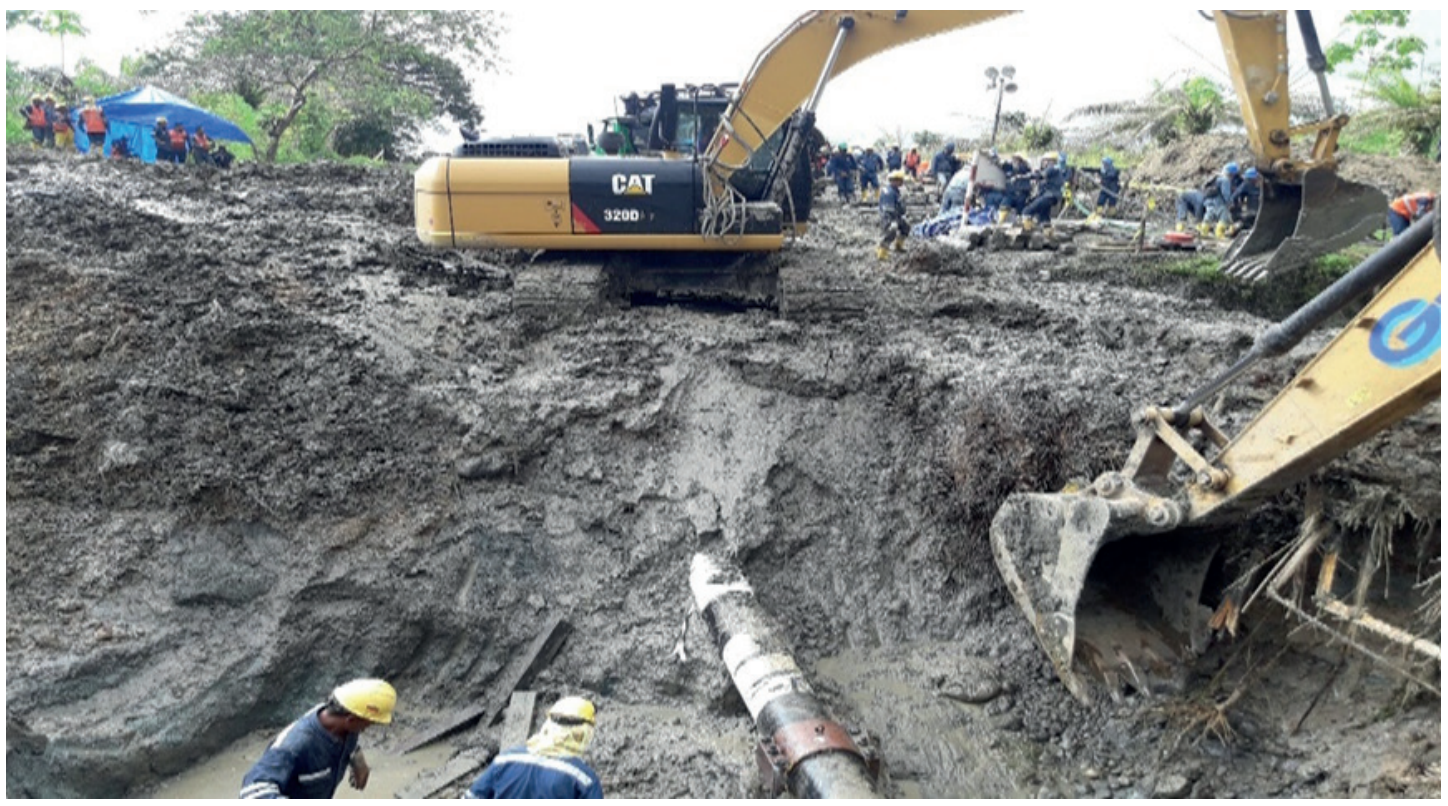

Figure 1. Esmeraldas-Santo Domingo Pipeline Rupture, Ecuador.

Source: Petroecuador.

ways to reduce unwanted incidents affecting nearby communities, hydrocarbon transport lines, the environment, and the right-of-way through which the pipelines containing hazardous products pass should be continued.

\section{METHODOLOGY}

In the investigation of pipeline failures with the quantitative data collected by the internal and external inspection, by using the MLF tool at $2.5 \mathrm{~km} / \mathrm{h}$, the risk determinants were identified from $\mathrm{km} 0$ to $\mathrm{km} 164$ of Petroecuador's Esmeraldas-Santo Domingo Pipeline. Applied research was developed for knowledge acquisition and experimental design of independent variables manipulation. The effects produced by the dependent variables were observed.

It is useful to have in mind that the new paradigm can change many habits, culture and practices. Several hidden properties appear when compared with a classical approach and they converge in a new risk control management paradigm that the technical parties are interested in applying (Massotte \& Corsi, 2015).

1. Exploratory: failure determinants are identified and monitored to find phenomena with high and low probability of cumulative risks (micro and macro cumulative risk).
2. Descriptive: specification of criticality determinants in the origin of the risk.

3. Correlational: associate the variables of the conformity and management status.

4. Explanatory: understand the uncertainty phenomenon and/or find management as a result of minimizing the impacts of failures, leaks and spills, and environmental contamination.

There are several types of surveys: external inspection, direct assessment or inline inspections using magnetic flux leakage (MFL) or ultrasonic equipment. All of them generate detailed information about the condition of the pipeline and the extent of anomalies and corrosion. This information guides the operation of the pipelines or defines maintenance activities (Adam \& Davis, 2009).

\section{Identification and risk assessment}

When comparing information about substandard actions and conditions, the cause-effect relationship of the risk is determined at the source, medium or receiver between the pipeline and the rights-of-way.

It was found that more accidents are caused by landslides in the Trans-Ecuadorian Oil Pipeline system and polyducts with quantitative properties related to the mechanical criticality of soil geological 


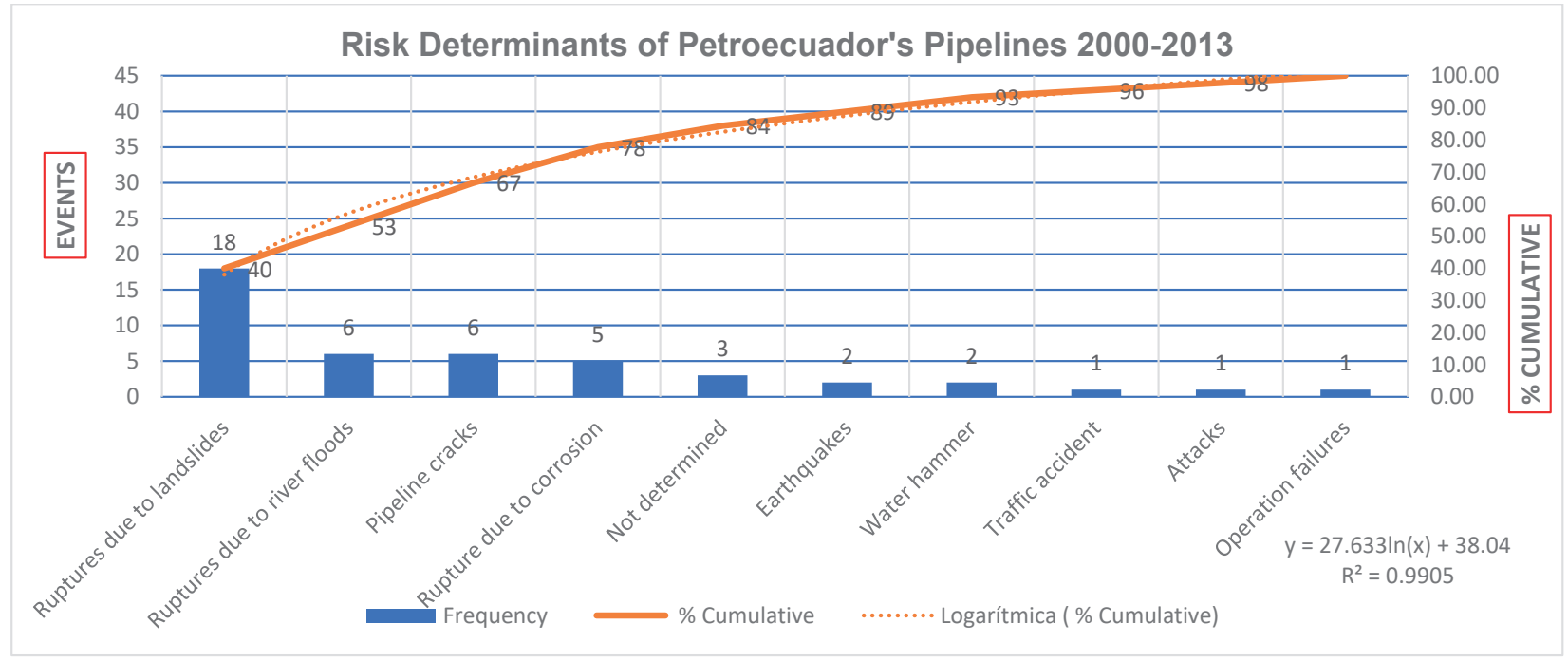

Figure 2. Risk determinants and failure probability, leakage and spill of hydrocarbons. Source: Petroecuador.

faults criticality, one of the reasons for hydrocarbon leakage. This can be seen in Figure 2.

According to Bai and Bai (2014), each pipe segment has its own risk, such as the production of failure probability and consequence of failure.

The high risk substandard conditions identified along the $164 \mathrm{~km}$ right-of-way (ROW) of the Esmeraldas-Santo Domingo Pipeline and oil pipeline are:

- Landslides and/or superficial ground movements.

- Landslides and/or deep ground movements.

- Land subsidence.

- Surface faulting and jointing in the rocky substrate.

- Fluvial erosion in the soft layers located on the margins of the ROW and the collapse of more resistant layers.

- Erosion sites that activate slopes and cause collapsed retaining walls.

- Signaling points of pipe dragging due to landslides-burial.

- "Shielding-piledriving-fault" point.

- Landforms along the right-of-way and their seismic influences.

- Altimetry profile of the pipeline and right-of-way (mapping alternatives routes).
- Population rate.

- Interference with property boundaries, streets and roads, garrisons and all data that helps to clearly define the right-of-way and the identification of faults and joints.

- Incorporation of incidence maps of the region; topographic, hydrographic, geologic, etc.

- Development of the altimetric profile, populated areas and pipe valves.

- Aerial photographs may be convenient if no maps or charts are available.

- Geotag and sectorial analysis.

- Identifying the influence of the passage of the pipelines according to the sectorial division by the National Military Geographic Institute of Ecuador.

- Determination of the land use maps, normally edited by the National Institute of Statistics, Geography and Informatics (INEGI). These maps will make it possible to define possible effects on the proposed or installed right-of-way.

\section{The risk control management model}

The model specifies and uses the integrity and reliability of pipelines through line maintenance; it emphasizes the sustainability of hydrocarbon transportation and right-of-way. 
- Integrity and reliability. Soil characterization of the right-of-way.

- Identifies the type of soils to improve the conductivity of the pipeline cathodic protection.

- Eddy current interference points that affect the external integrity of the pipeline.

- Internal cleaning tools.

- Inspection tools (transmitter/receiver, ultrasound, magnetic flux leakage).

- Soil/pipeline resistivity results.

- Hydrodynamic analysis on right-of-way.

- Hydrocarbon leakage control points.

- Magnetic flux monitoring through impressed current system.

- Dosage and injection of corrosion inhibitor.

- Monitoring of wall thickness loss and their progress over time.

- Reviews of analysis of field data result reports.

It also leads to the detailed organizational and technical preparation of the pipeline, which is required to enable the launch and receipt of intelligent cleaning tools, which guarantees their safe passage and the most accurate data on the state of corrosion (Timashev \& Bushinskaya, 2016).

\section{ON-OFF potential and Native potential on right- of-way}

- Test the chloride content of the soil.

- Review and check the soil resistivity.

- Inspect the proximity to high voltage power lines.

- Inspect potential variables between pipes.

- Intervene in soils affected by eddy currents.
- Inspect crossings with other electrical or structural interconnection lines.

- Review records going back three years.

- Verify reading of $850 \mathrm{mV}=>$ ON potential <= $2100 \mathrm{mV}$ (quarterly).

- Verify ON potential reading of $<850 \mathrm{mV}$, verify testing procedure.

- Verify compliance with the $100 \mathrm{mV}$ criterion.

We could quantify the losses present in any hydrocarbon transportation system, such as the costs of service interruption, the cost of lost product, the cost of cleaning, among others. The consequences are sometimes grouped into direct and indirect categories, where the direct costs are inclusive of integrity and influence the processes of risk control in the face of hydrocarbon leaks (Muhlbauer, 2004).

\section{Results obtained from internal and external ins- pection}

The inline magnetic flux leakage (MFL) tool was launched. Internal and external corrosion anomalies were observed, as can be seen in the results of the integrity coefficient as an indicator (see Figure 3 ). It was determined that there are major failures due to external corrosion, but there is a high prevalence of significant risk of cumulative anomalies at coefficients < 1 , with 1 being a critical value in relation to the dip width of the corrosion anomaly and the design pressure rating with the maximum allowable operating pressure calculated based on the discharge pressure of the constant flow.

Corrosion can be defined as the deterioration of a metal due to chemical or electrochemical reactions between the metal and its environment. The tendency of a metal to corrode depends on the environment and the type of metal. The presence of

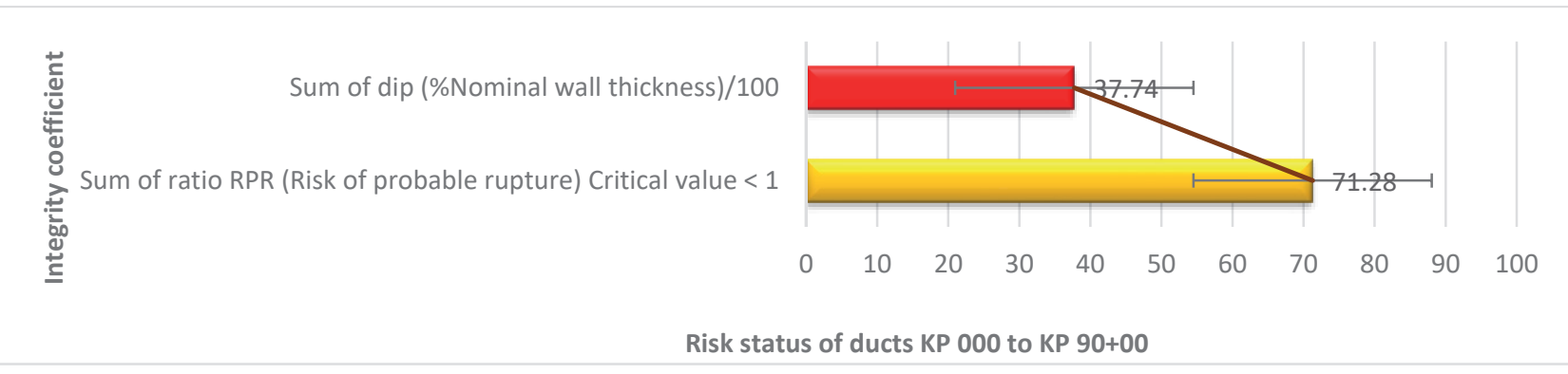

Figure 3. Status of corrosion risk control of Esmeraldas-Santo Domingo Pipeline, 2014 (kp 000 to kp 90). Source: Petroecuador. Internal and external inspection Esmeraldas-Santo Domingo Pipeline 2014. 
carbon dioxide $\left(\mathrm{CO}_{2}\right)$, hydrogen sulphide $\left(\mathrm{H}_{2} \mathrm{~S}\right)$ and free water in the production fluid can cause serious corrosion problems (Bai \& Bai, 2014).

The status of risk control of the Esmeraldas-Santo Domingo Pipeline in 2017 is presented. A significant improvement can be observed in 2017 (see Figure 4) in relation to 2014 (see Figure 3).

\section{Useful and sustainable}

The management model makes the structure safe along the operations and links it to the development and innovation of proposals aimed at reducing the losses generated by the pipelines; thus, it seeks to identify the probability of failure by analyzing the conditions and actions that interfere along the right-of-way of the 164 kilometer Esmeraldas-Santo Domingo Pipeline, for which a sample is taken into account (internal risk from kp 050 to kp 96).

From the sample, the following were developed: services of technical comparison with operation and control standards; regulatory requirements (see Table 1); codes for inspection, maintenance, qualification, repair and alteration practices; predictive

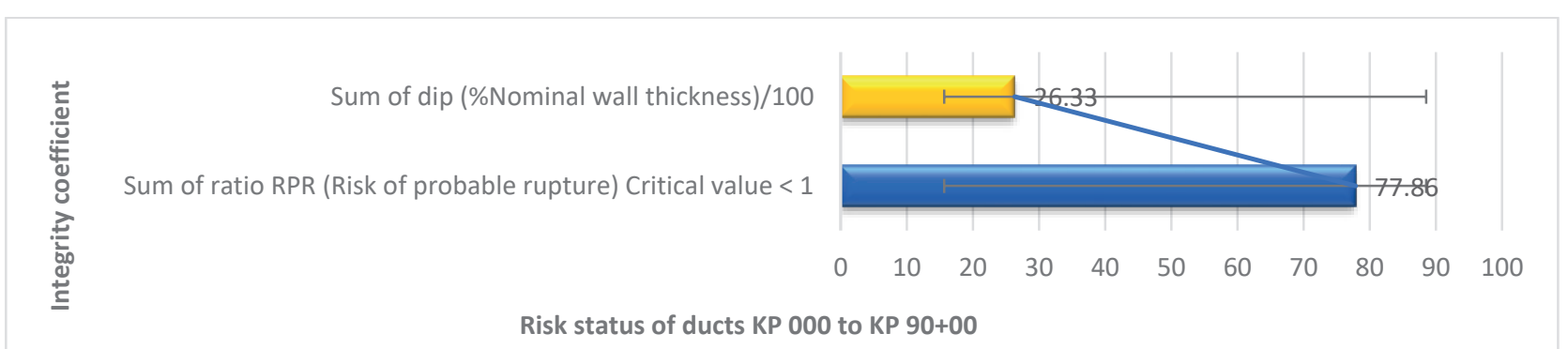

Figure 4. Status of corrosion risk control of the Esmeraldas-Santo Domingo Pipeline, year 2017 (pk 000 to pk 90). Source. Petroecuador. Internal and external inspection of Esmeraldas-Santo Domingo Pipeline 2017.

Table 1. Facilities and their operational conformity.

\begin{tabular}{|c|c|c|c|c|c|}
\hline \multicolumn{6}{|c|}{ Level of management: Risk Control $(\mathrm{N}+, \mathrm{N}-; \mathrm{C}) /$ Conformity status } \\
\hline $\begin{array}{c}\# \\
\text { Fac. }\end{array}$ & \multicolumn{3}{|c|}{$\begin{array}{l}\text { Facilities: Conformity status / integrity } \\
\text { and reliability }\end{array}$} & \multicolumn{2}{|c|}{ Operating Units } \\
\hline 1 & \multicolumn{3}{|l|}{ Pipelines } & \multirow{4}{*}{\multicolumn{2}{|c|}{$\begin{array}{l}\text { OMU 1: Line maintenance of the hydrocarbon transport system } \\
\text { OMU 4: Integrity and reliability of hydrocarbon transport } \\
\text { OMU 7: Physical security "Military patrols and civilian personnel" } \\
\text { OMU 5: Geomatics for the right-of-way and hydrocarbon transport pipelines }\end{array}$}} \\
\hline 2 & Rectifiers & & & & \\
\hline 3 & \multirow{2}{*}{\multicolumn{3}{|c|}{ Right-of-way }} & & \\
\hline 4 & & & & & \\
\hline 5 & \multicolumn{3}{|c|}{ Pumping station } & \multirow{3}{*}{\multicolumn{2}{|c|}{$\begin{array}{l}\text { OMU 2: Operations I } \\
\text { OMU 3: Operations II } \\
\text { OMU 6: Laboratory }\end{array}$}} \\
\hline 6 & \multicolumn{3}{|c|}{ Station tank farms } & & \\
\hline 7 & \multicolumn{3}{|c|}{ Manifold launcher tool } & & \\
\hline 8 & \multicolumn{3}{|c|}{ In-line flow control valve } & \multicolumn{2}{|c|}{ OMU 8: Electromechanical Maintenance of the pumping station } \\
\hline C & NC_neg & NC_pos & $\% \mathrm{C}$ & Period: & Units: integrity analysis \\
\hline 34 & 0 & 0 & & \multirow{9}{*}{ Month/year } & 34 \\
\hline 42 & 0 & 0 & & & 42 \\
\hline 8 & 0 & 0 & & & 8 \\
\hline 24 & 0 & 0 & & & 24 \\
\hline 6 & 0 & 0 & & & 6 \\
\hline 13 & 0 & 0 & & & 13 \\
\hline 11 & 0 & 0 & & & 11 \\
\hline 8 & 0 & 0 & & & 8 \\
\hline 146 & 0 & 0 & & & 146 \\
\hline
\end{tabular}

OMU: operation management units.

Source: Prepared by the author. 
welding; event practices; recommended practices; safe practices; instrumentation practices; destructive testing; non-destructive testing; maintenance of impressed current deep groundbeds; practice of manual pipe welding to determine resistance and methods to evaluate the acceptance of defects in metal structures.

Such parameters should be identified for each gas pipeline and monitoring of chemical and physical mitigation methods should be included (Kermani \& Chevrot, 2012).

The performance of operational integrity is identified, acknowledging conformity by the percentage of compliance with standards in relation to the technical comparison instrument used to verify compliance with the operational plan focusing on major nonconformities, minor nonconformities and conformity of the control of risks in the reliability of hydrocarbon transportation.

Risk Longitudinal Analysis and Pipeline Intervention (ALRI, for its Spanish acronym) conformity formula

The ALRI conformity formula identifies the status of management at the facilities:

$$
\mathrm{C}=\left(\mathrm{C} / \Sigma \mathrm{C}+\mathrm{N}_{-}+\mathrm{N}+\right) 1
$$

Where:

C: = Conformity; fulfilment of a requirement converted into activity.

$\mathrm{N}-=$ Minor nonconformity; lack of updating and implementation of procedure.

$\mathrm{N}+=$ Major nonconformity; lack of compliance with the procedure at the event control.

\section{$1=$ Constant}

The level of risk control management for 2014 is obtained, presenting a maximum non-permissible criticality level of 2.654, which marks its state of vulnerability of the topography to faults, leaks and major spills with respect to the insecurity estimated by the operating units of the oil, polyducts and gas pipelines. The comparison instrument is used for reference (see table 2).

The concentration of independent variables generates determinants of failure modes in the factors adjacent to the design of the implementation of the hydrocarbon transportation facilities system, in which there is a probability of events (cumulative risks) that trigger undesired events with a risk level of 2.51, which represents unsafe conditions (low validity) during the period of 2014. This indicates an unsatisfactory management on a 10-point basis for perfect management validity, which is reflected by 8 components (operating units), observed in Table 3.

The technical comparison matrix improved the reliability and integrity of the operating units; in addition, it managed to consolidate the management progressive and significantly influence the end of 2017 on the basis of 6.08 , in relation to the verification of technical compliance of the soil, pipelines and flow through pipes. This had an impact on the permanent management to achieve the integrity and reliability of safe hydrocarbon transportation and minimize the environmental impacts generated, such as macro-cumulative risks.

The risk level for compliance with technical comparison applied to the correlation of the years 20142017 by the number of analysis units with corporate responsibility for hydrocarbon transport can be seen in Figure 5.

Table 2. Units of risk management control in terms of analysis units.

\begin{tabular}{|l|l|}
\multicolumn{1}{|c|}{ Operating units for polyducts, oil and gas pipelines } & \multicolumn{1}{|c|}{ Analysis units } \\
\hline OMU 1: Line maintenance of the hydrocarbon transport system & 34 \\
\hline OMU 2: Operations I & 13 \\
\hline OMU 3: Operations II & 6 \\
\hline OMU 4: Integrity and reliability of hydrocarbon transport & 42 \\
\hline OMU 5: Geomatics for the right-of-way and hydrocarbon transport pipelines & 24 \\
\hline OMU 6: Laboratory & 11 \\
\hline OMU 7: Physical security "Military patrols and civilian personnel” & 8 \\
\hline OMU 8: Electromechanical Maintenance of the pumping station & 8 \\
\hline Total & 146 \\
\hline
\end{tabular}

Source: Prepared by the author. 
It is evident that the level of risk control is significant at $5-10 \%$ at the end of 2017 .

\section{Risk management control level/operating risk formula for technical comparison}

$$
\begin{gathered}
\mathrm{Ng}=\text { VUG1 }+ \text { VUG2+VUG3+... VUG8 } \\
\mathrm{Ng}=\left(\mathrm{Na} / \mathrm{Va}{ }^{*} \mathrm{Vn}\right) * 10
\end{gathered}
$$

Where:

VUG $=$ Total value of operational corrective actions.

$\mathrm{Na}=$ Number of activities.

$\mathrm{Ng}=$ Level of risk management.

$\mathrm{Vn}=$ Nominal value .

$\mathrm{Va}=$ Value of analysis units.

\section{RESULTS}

\section{Result 1}

The traditional management approach towards a change of management based on identification, monitoring, control, measurement and disclosure of risks allows us to maintain continuity of the hydrocarbon transport business with a reduction of line leakage resulting in environmentally friendly operations.

The table 4 shows the difference between the traditional model and the new model approach to risk control management and assessment of unplanned conditions in hydrocarbon pipelines. According to the trend, the Esmeraldas-Quito Pipelines show a reduction from 3.5 events per year to 1 event per year.

Table 3. Risk control management indicators on a 1-10 basis.

\begin{tabular}{|c|c|c|c|c|}
\hline \multirow{2}{*}{ Management units } & $\mathbf{2 0 1 4}$ & $\mathbf{2 0 1 5}$ & $\mathbf{2 0 1 6}$ & $\mathbf{2 0 1 7}$ \\
\cline { 2 - 5 } & MU score/10 & MU score /10 & MU score /10 & MU score /10 \\
\hline OMU 1 & 3.29 & 4.14 & 4.82 & 6.35 \\
\hline OMU 2 & 3.88 & 4.10 & 4.74 & 7.50 \\
\hline OMU 3 & 1.53 & 2.50 & 3.06 & 8.00 \\
\hline OMU 4 & 3.17 & 3.33 & 4.68 & 6.27 \\
\hline OMU 5 & 1.67 & 2.75 & 3.44 & 3.11 \\
\hline OMU 6 & 1.00 & 1.82 & 3.48 & 8.00 \\
\hline OMU 7 & 3.75 & 4.17 & 6.80 & 3.78 \\
\hline OMU 8 & 1.80 & 2.76 & 2.85 & 6.08 \\
\hline Total, MU Risk & 2.51 & 3.20 & 4.23 & \\
\hline
\end{tabular}

Source: Prepared by the author.

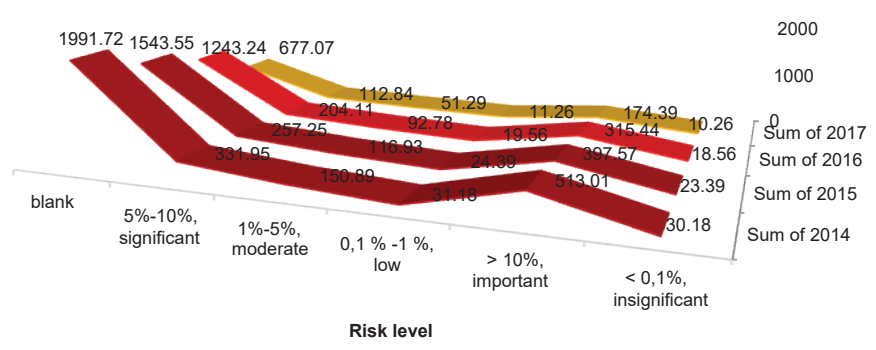

2000

of 2016

of 2015

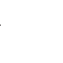

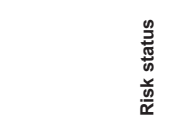

Figure 5. Status of the level of risk control of Esmeralda-Santo Domingo Pipeline in 2014-2017.

Source: Prepared by the author. 
Figure 6 shows the result obtained in the implementation of the Esmeraldas-Santo Domingo Pipeline Management Model at the end of 2017.

In the sense of effective risk management, results are guaranteed in accordance with the strategic objectives of the Petroecuador organization; with this vision, the integral management of risk control becomes the key factor in the success of reducing line losses and environmental contamination. Management systems are "proven structures for the management and continuous improvement of policies, procedures and processes of the organization" (SIGWEB, 2016). These systems can be individual or complex interactions.

\section{Result 2}

Installed capacity of $30,660,000$ Bbls/year. The yield at the end of the period 2014 to 2017 in barrels of hydrocarbons transported by the Petroecuador Esmeraldas-Santo Domingo Pipeline with significant trend in the year 2018:

Year 2014: 22,505,273 Bbls/year transported, representing $72 \%$ of expected production. (Start of risk control management).
Year 2017: 24,077,474 Bbls/year transported, representing $78.53 \%$ of expected production. (Expected results).

Year 2018: 25,523,034 Bbls/ year transported, representing $83.25 \%$ of expected production. (Reliability and integrity trend).

Risk control management model guidelines:

a. Provides criteria for intervention in the face of opportunities for improvement and command of risk control in the operational management units and conformity with facilities in hydrocarbon transport processes; which will depend on strategies and technical methods for intervention, such as business sustainability plans in relation to expected results (zero claims).

b. Requires a risk map which shows areas exposed to leaks with fire and explosion; consequently, it establishes the maximum acceptable risk, as well as the unacceptable area.

c. Uses the measurement of risk category-monitoring with probability to cause damage to the

Table 4. Conformity analysis in hydrocarbon transport pipelines.

\begin{tabular}{|c|c|c|c|}
\hline Row labels & Sum of C & Sum of NC_neg & Sum of NC_pos \\
\hline 2014 & 16 & 41 & 89 \\
\hline 2015 & 40 & 29 & 77 \\
\hline 2016 & 65 & 37 & 15 \\
\hline 2017 & 101 & 22 & 2 \\
\hline Year & 2 & 4 & 227 \\
\hline Grand total & 224 & 133 & \\
\hline
\end{tabular}

Conformity, NC-neg = Minor nonconformity; NC_pos = Major nonconformity.

Source: Prepared by the author.

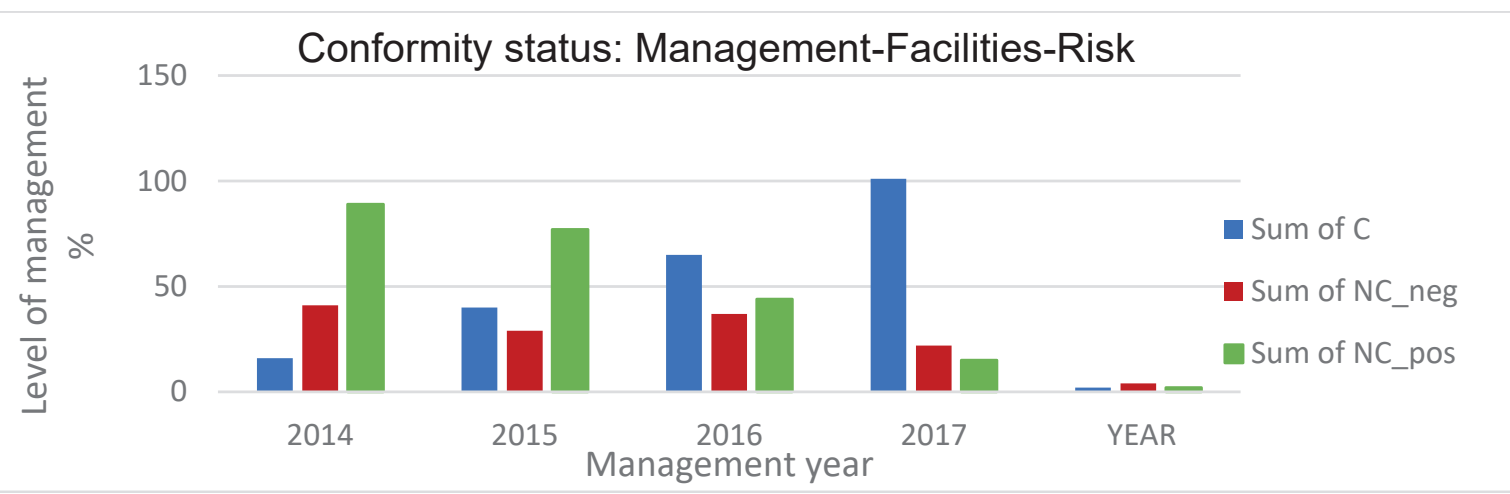

Figure 6. Risk management control concerning functional conformity of the Esmeralda-Santo Domingo Pipeline in 20142017, Petroecuador.

Source: Prepared by the author. 
process of hydrocarbons transportation by pipeline and right-of-way.

d. Uses the projection of the loss level before the monitoring-measurement of permanent control in the source, the medium and the receiver that originate losses by cause and effect of the materialization of the incident.

e. Specifies the projection mechanisms for maximum contingency coverage against the risk of hydrocarbon leakage.

f. Uses, along the right-of-way, the permanent early warning coverage of danger and risk with probability of accidents in populations near the transport of hydrocarbons.

g. Defines and considers performance measures to be risk adjusted identification along the rightof-way and pipelines.

\section{Result 3}

The structures of the management model design were consolidated to obtain the final product of the organization in a software program (see Figure 7). The handling of applications developed and incorporated into the operational units of the risk control mandate is presented, operating from the base of the corporate system with entry and validation of the information executed in the field.

The risk control programmer can help quantify cost and safety equilibrium at the design stage, and also identify policies and schedules for cost-effective testing, preventive maintenance, spare parts provision, redundancy allocation and replacement of working parts to keep complex systems operating as intended throughout their design life (Cox, 2009).

\section{DISCUSSION}

The risk control management model for oil companies makes it possible to influence insurance policy brokers in the oil industry. When taking possession of assets, the aim is not to eliminate risks, but rather the efficient management of risks by the insurance company, for which it is necessary to identify, measure and control risks; in this way, risk prevention and the model established in the operational management units work in an integrated manner and not separately.

\section{CONCLUSION}

Sufficient evidence was found at the risk status levels to assert that the corporate event control management model of hydrocarbon transportation with

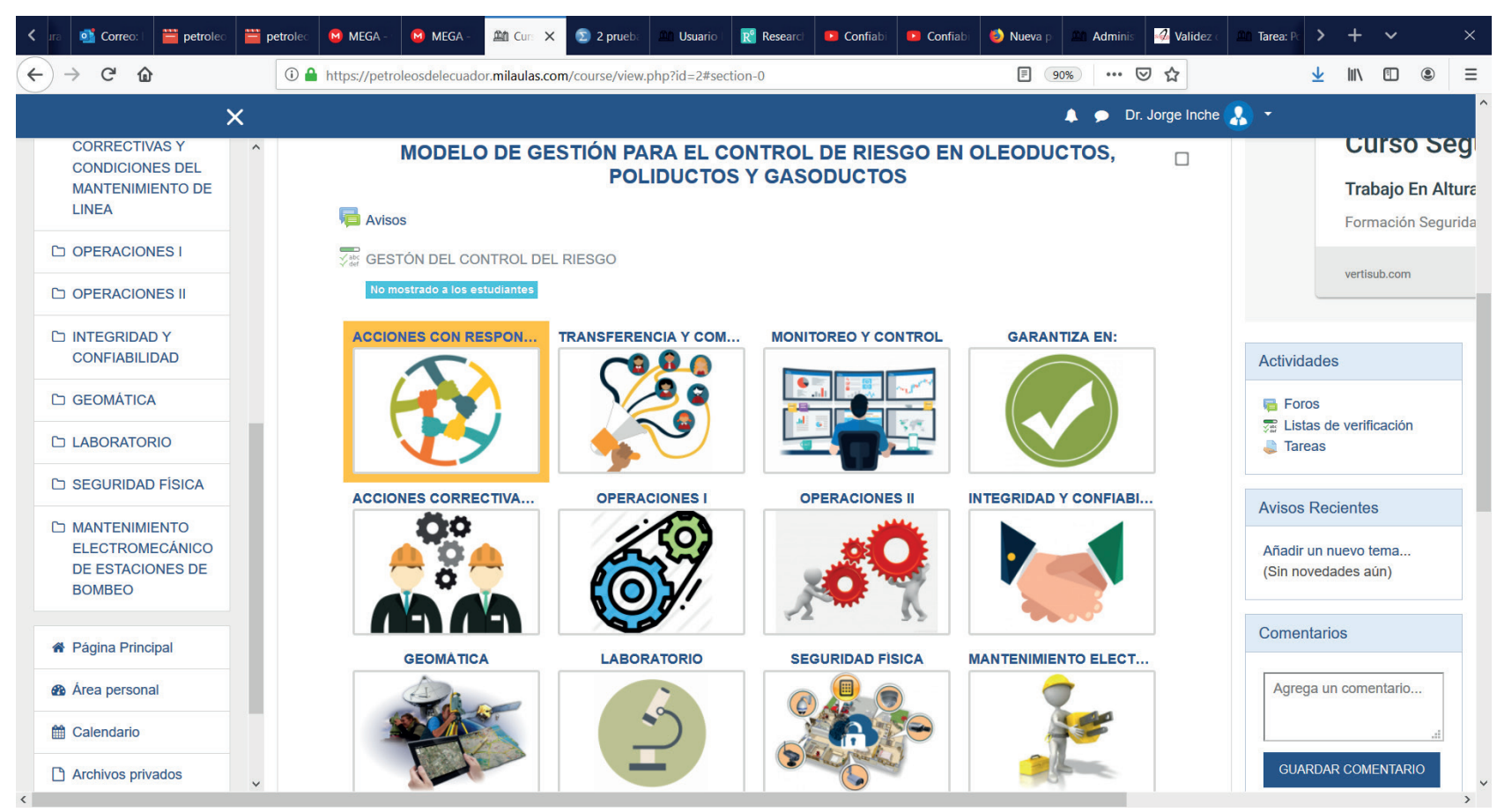

Figure 7. Operational management model from the corporate platform.

Source: Prepared by the author. Software model of risk control management of hydrocarbon transport. 
scores of the operational management units significantly influences the compliance percentages of the facilities (hydrocarbon flow, pipeline and rightof-way soil).

- Corrosion was controlled in compliance with the integrity and reliability standards of the pipelines (external and internal anomalies); with the RPR critical value $<1$ the risk level status of the ducts was controlled and, as a result, production increased.

- In 2014, the maximum allowable operating pressure was 1050 psi and $2600 \mathrm{Bbls} /$ hour; in 2018, the load rose to 1200 psi and $3200 \mathrm{Bbls} /$ hour for gasoline and 3000 Bbls/hour for diesel oil, so that the estimated demand projection was met, using the installed capacity.

- Environmental damage was minimized and the installed capacity of hydrocarbon transportation for the period 2014 was used, which was $72 \%$; for 2017 , it was $78.53 \%$; and for 2018 , it was more than $83.25 \%$.

\section{ACKNOWLEDGMENT}

The author is deeply grateful to the Petroecuador Esmeraldas-Santo Domingo-Quito Pipeline operational units for access to facilities in favor of research and development, which we permanently link to risk prevention, with a view to reducing environmental impacts and improving the reliability and integrity of the operating processes of hydrocarbon transportation. This research is conducted in compliance with the standardized technical comparison that, in addition, constitutes a friendly practice with the environment and communities surrounding the right-of-way.

\section{REFERENCES}

[1] Adam, S. \& Davis, K. (2009). Pipeline Geomatics. Practice and Innovation. Fairfield, United States: American Society of Mechanical Engineers.

[2] Bai, Y. \& Bai, Q. (2014). Subsea Pipeline Integrity and Risk Management. Massachusetts, United States: Gulf Professional Publishing.
[3] Cox, A. (2009). Risk Analysis of Complex and Uncertain Systems. New York, United States: Springer.

[4] Guy, P. \& Elwany, M. H. (2007). Safety, Reliability and Risks Associated with Water, Oil and Gas Pipelines. Heidelberg, Germany: Springer.

[5] Kermani, B.\& Chevrot, T. (2012). Recommended Practice for Corrosion Management of Pipelines in Oil and Gas Production and Transportation. Leeds, United Kingdom: Maney Publishing / European Federation of Corrosion.

[6] La Hora (February 26, 2005). Hace siete años Esmeraldas vivió una noche de terror. La Hora. Retrieved from https://lahora.com. ec/noticia/1000310432/hace-siete-aosesmeraldas-vivi-una-noche-de-terror.

[7] La Hora (April 9, 2013). Derrame de 5.500 barriles de crudo en Esmeraldas. La Hora. Retrieved from https://lahora.com.ec/ noticia/1101489994/derrame-de-5500-barrilesde-crudo-en-esmeraldas.

[8] Massotte, P. \& Corsi, P. (2015). Operationalizing Sustainability. London, United Kingdom: Wiley / ISTE.

[9] Muhlbauer, W. K. (2004). Pipeline Risk Mangement Manual. Ideas, Techniques and Resources. Massachusetts, United States: Elsevier.

[10] Organismo de Evaluación y Fiscalización Ambiental (2016). Resolución Directoral N. ${ }^{\circ}$ 12-2016-OEFA/DS. Informe N. ${ }^{\circ}$ 307-2016OEFA/DS-HID. Retrieved from http://www. oefa.gob.pe/wp-content/uploads/2019/03/ RESOLUCION-N012-2016-DS.pdf.

[11] SIGWEB (2016). Ventajas de los Sistemas de Gestión de Seguridad y Salud en Trabajo. SIGWEB. Retrieved from http://www.sigweb. cl/2016/10/20/sistemas-integrados-de-gestion.

[12] Timashev, S. \& Bushinskaya., A. (2016). Diagnostics and Reliability of Pipeline Systems. Cham, Switzerland: Springer. 